\title{
醌式杂环化合物的设计、合成及应用性能研究进展
}

\author{
蔡金芳 ${ }^{a}$ 江 华*,a,b 崔志华 $a, b$ 陈维国 ${ }^{a, b}$ \\ ( ${ }^{a}$ 浙江理工大学 生态染整技术教育部工程研究中心 杭州 310018) \\ ( ${ }^{b}$ 浙江理工大学 先进纺织材料与制备技术教育部重点实验室＼cjkstart杭州 310018)
}

\begin{abstract}
摘要 醌式杂环(噻吩、吡咯、呋喃等)分子具有结构刚性、最高占据分子轨道(HOMO)/最低未占据分子轨道(LUMO) 能级低、能级带隙窄和摩尔消光系数高等特点. 醌式分子因其结构平面性特点, 分子间作用力较强, 因而分子间电荷传 输能力强. 目前, 醌式杂环分子已成为有机半导体材料领域特别是有机场效应晶体管领域的研究热点. 根据醌式杂环 分子的结构特点，以端基为分类依据，综述了近年来醌式杂环化合物在分子设计、合成及应用性能等方面的研究进展， 并展望了醌式杂环分子的发展前景.
\end{abstract}

关键词＼cjkstart醌式杂环化合物; 设计与合成; 性质与应用; 有机半导体材料; 有机场效应晶体管

\section{Research Progress in Design, Synthesis and Application for Quinoidal Heterocyclic Compounds}

\author{
Cai, Jinfang ${ }^{a} \quad$ Jiang, Hua ${ }^{*, a, b}$ Cui, Zhihua ${ }^{a, b}$ Chen, Weiguo ${ }^{a, b}$ \\ ( ${ }^{a}$ Engineering Research Center for Eco-dyeing and Finishing of Textiles, Ministry of Education, \\ Zhejiang Sci-Tech University, Hangzhou 310018) \\ $\left({ }^{b}\right.$ Key Laboratory of Advanced Textile Materials and Manufacturing Technology, Ministry of Education, Hangzhou 310018)
}

\begin{abstract}
Quinoidal heterocyclic (thiophene, pyrrole, furan, etc.) molecules have the characteristics of rigid backbone, low highest occupied molecular orbital (HOMO)/lowest unoccupied molecular orbital (LUMO) level, narrow band gap and high molar extinction coefficient, and so on. Because of their planar structures, quinoidal molecules usually have strong intermolecular charge transfer ability due to the strong intermolecular interaction. Up to now, quinoidal heterocyclic molecules have become a research hotspot in the field of organic semiconductor materials, especially in the field of organic field effect transistors. According to the structural features of quinoidal heterocyclic molecules and the classification basis of terminal groups, the research progress of quinoidal heterocyclic compounds in molecular design, synthesis and application in recent years is reviewed, and the development of quinoidal heterocyclic molecules is prospected.

Keywords quinoidal heterocyclic compound; design and synthesis; property and application; organic semiconductor material; organic field effect transistors
\end{abstract}

近几十年来, 醌式杂环化合物因其具有结构平面刚 性、最低未占据分子轨道(LUMO)能级低、最高占据分 子轨道(HOMO)//LUMO 能级带隙窄及吸收强度大等特 点, 作为有机半导体材料而受到广泛关注 ${ }^{[1-3]}$. 从结构 上看，可将醌式分子分为醌式杂环母体和端基两部分. 其中, 醌式杂环母体提供醌式分子的主要性质, 如分子 平面性和吸收光特性等; 端基则起到稳定醌式状态及阻 止醌式结构恢复芳香结构的作用. 常见的端基包括二氰
基亚甲基、(烷氧基)羰基氰基亚甲基、酰基氰基亚甲基、 二苯基亚甲基、亚芴基和苯醌基等. 通常吸电性端基较 供电性端基更有利于稳定醌式杂环结构. 当端基无法稳 定醌式结构时，醌式结构往往以双自由基形式存在. 醌 式结构的双自由基物质同样也受到众多科学家的关注. 鉴于此，本文根据醌式杂环结构的特点，将其分为二氰 基亚甲基、(烷氧基)㷇基氰基亚甲基、酰基氰基亚甲基、 二苯基亚甲基、亚芴基和苯醌基等醌式杂环化合物，对

* Corresponding author. E-mail: jh@zstu.edu.cn

Received September 15, 2019; revised October 14, 2019; published online November 1, 2019.

Project supported by the Public Welfare Technology Research Project of Zhejiang Province (No. LGG18B060003), the National Natural Science Foundation of China (Nos. 21808210, 51673176) and the Fundamental Research Funds of Zhejiang Sci-Tech University (No. 2019Q018).

浙江省公益技术研究计划(No. LGG18B060003)、国家自然科学基金(Nos. 21808210, 51673176)和浙江理工大学基本科研业务费专项资金(No. 2019Q018)资助项目. 
各类醌式杂环化合物的研究进展进行了综述.

\section{1 二首基亚甲基醌式杂环化合物}

二氰基亚甲基醌式杂环化合物是以二㲵基亚甲基 为端基的一类醌式分子, 可以看作是四氰基对二甲基苯 醌(TCNQ, 1) 的衍生物, 是最早出现的一类醌式杂环化 合物. 基于 TCNQ 单晶的场效应晶体管的电子迁移率可 达 $0.5 \mathrm{~cm}^{2} \cdot \mathrm{V}^{-1} \cdot \mathrm{s}^{-1}$, 显示出了 TCNQ 作为 $\mathrm{n}$ 型半导体材 料的巨大潜力 ${ }^{[4]}$. 但 TCNQ 及其衍生物存在溶解性差、 结构难于扩展和制膜困难等缺点, 进而催生出二氰基亚 甲基醌式噻吩衍生物。<smiles></smiles>

最初, 制备二氰基亚甲基醌式噻吩衍生物的方法是 以二溴代噻吩为底物, 四氧基环氧乙烷为端基化试剂, 在 1,2-二溴乙烷中回流一定时间(Eq. 1) ${ }^{[5 \sim 8]}$. 采用该方 法可以制备具有 1 3 个噻吩单元的简单醌式分子. 不 过，该方法存在反应温度高 $\left(>130{ }^{\circ} \mathrm{C}\right)$ 、反应时间长、 收率低(10\% 40\%)和底物拓展受限等缺点, 未能得到 广泛应用。

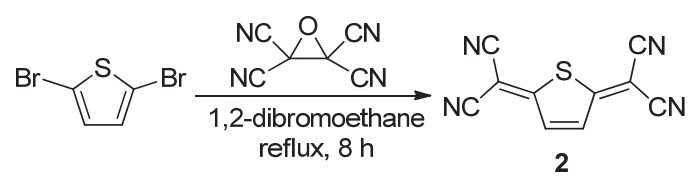

1984 年, Takahashi 课题组 ${ }^{[9]}$ 报道了丙二腈负离子在 钯催化剂的作用下与卤代芳香化合物作用生成二氧基 甲基芳香化合物的碳一碳偶联反应, 该反应也被称之为 Takahashi 反应. 随后, Ogura 等 ${ }^{[6]}$ 应用 Takahashi 反应在 二溴代联噻吩上对称地接入二氧基甲基, 再在溴水的作 用下经氧化反应生成二氰基亚甲基醌式联噻吩化合物 $\mathbf{3}$ (Scheme 1). 与以四氰基环氧乙烷为端基化试剂的合成 方法相比, 采用 Takahashi 反应的合成方法极大地提高 了反应收率 $(>60 \%)$, 且底物拓展适用性良好.<smiles>CC(C#N)c1ccc(-c2ccc(C(C)C(C)C)s2)s1</smiles><smiles>N#CC(C#N)=c1ccc(=c2ccc(=C(C#N)C#N)s2)s1</smiles>

图式 1 Takahashi 反应合成二氰基亚甲基醌式噻吩 Scheme 1 Synthesis of dicyanomethylene quinoidal thiophene by Takahashi reaction
另外，针对二噻吩并吡咯为母体的二氧基亚甲基醌 式化合物，文献还报道了另一种方法(Scheme 2). 2008 年, Rasmussen 等 ${ }^{[10]}$ 在研究三氰基乙烯基化合物时发现, 二噻吩并 $\left[3,2-b: 2^{\prime}, 3^{\prime}-d\right]$ 吡咯在与四氰基乙烯(TCNE)反应 时，除生成主要的三氰基乙烯基化合物外，还得到了部 分二氰基亚甲基醌式化合物 4(收率 10\%). Marder 等 ${ }^{[11]}$ 在此基础上采用富电性底物以 $29 \%$ 的收率得到醌式化 合物 5 , 使得该法也成为一种构建二㲵基亚甲基醌式结 构的可选途径.

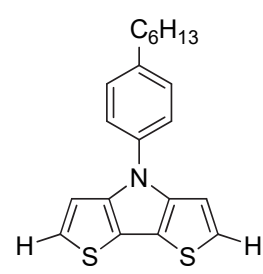

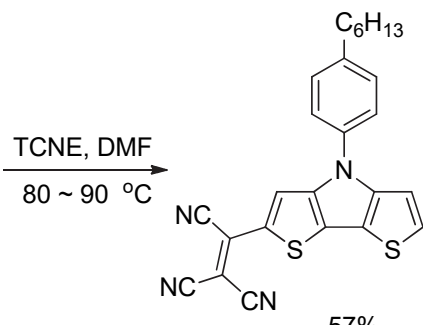<smiles>CCCCCCc1ccc(-n2c3cc(C(C#N)=C(C#N)C#N)sc3c3sc(C(C#N)=C(C#N)C#N)cc32)cc1</smiles>

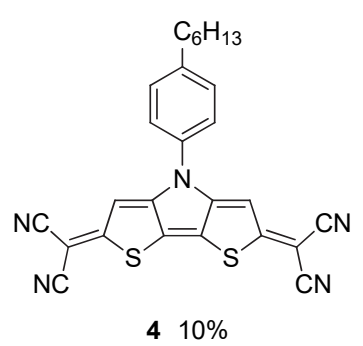

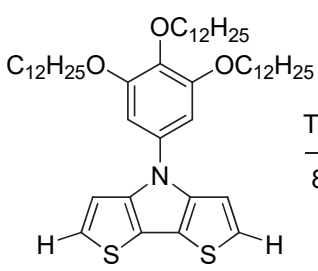
$\underset{80 \sim 110{ }^{\circ} \mathrm{C}}{\stackrel{\text { TCNE, DMF }}{\longrightarrow}}$

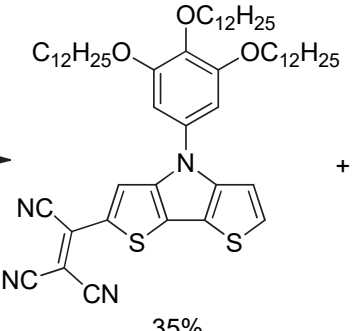

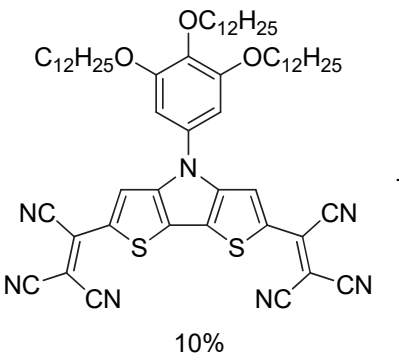

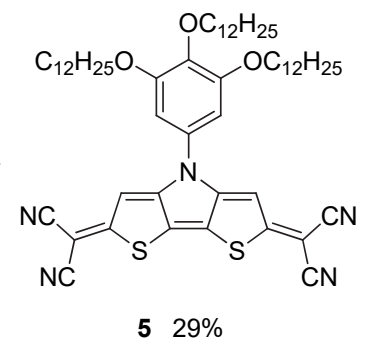

图式 2 以四氰基乙烯为端基化试剂合成醌式噻吩衍生物 Scheme 2 Synthesis of dicyanomethylene quinoidal thiophene by using tetracyanoethylene as terminal reagent

氰基由于强吸电子能力可以很好地稳定醌式结构, 二氰基亚甲基醌式杂环结构可以较容易地进行结构衍 生. 同时, 该类醌式分子普遍具有较低的 LUMO 能级 $(<-4.0 \mathrm{eV})$, 使其在 $\mathrm{n}$ 型有机场效应晶体管领域受到 
青睐. 目前, Takahashi 反应及氧化反应已成为构建二氰 基亚甲基醌式杂环分子的主要方法, 分子拓展的方式更 多地集中于设计醌式杂环母体. 二氧基亚甲基醌式噻吩 衍生物的母体拓展方法主要体现在以下四个方面.

增加噻吩单元的数量用以研究拓展的醌式 $\pi$ 共轭体 系与性能之间的关系 ${ }^{[12 ~ 20]} .2003$ 年, Frisbie 课题组 ${ }^{[12,13]}$ 报道了以 $3^{\prime}, 4^{\prime}$-二丁基-2,2':5',2"-联三噻吩为母体的二氧 基亚甲基醌式化合物 $\mathbf{6}$, 并首次将醌式噻吩化合物应用 于有机场效应晶体管中, 取得 $0.02 \mathrm{~cm}^{2} \cdot \mathrm{V}^{-1} \cdot \mathrm{s}^{-1}$ 的电子 迁移率. Otsubo 课题组 ${ }^{[15]}$ 以 5,5-二丁氧基- $4 H$-环戊烷并 $[c]$ 噻吩为噻吩单元, 成功地将醌式噻吩体系拓展至六 个醌式噻吩单元 $(\mathbf{7 a} \sim 7 \mathbf{f})$. 丁氧基有效克服了醌式分子 随体系增大而溶解性大幅降低的缺点. 随着噻吩单元的 增加, 化合物 $7 \mathbf{a} \sim 7 \mathbf{f}$ 在溶液中的吸收波长由可见光区 红移至近红外光区域. 同时发现在醌式分子 $7 \mathbf{e}$ 和 $7 \mathbf{f}$ 中 分别有 2.8\%和 29\%的分子以双自由基形式存在, 表明 此时二氰基亚甲基已难以维持稳定的醌式结构. 到目前 为止, 六个醌式寊噻吩单元已是二氧基亚甲基醌式噻吩 体系的极限. 为研究苯环对稳定醌式基态结构的作用, 2018 年, Aso 课题组 ${ }^{[20]}$ 报道了基于苯并 $[c]$ 噻吩结构单元 的醌式噻吩化合物 8 . 经计算, 化合物 8 的单重态双自 由基特征值 (y)为 0.56 , 而不含苯环的相似物的 $y$ 值为 0.75. 其最大吸收波长为 $978 \mathrm{~nm}$, 摩尔消光系数达到 $2.6 \times 10^{5} \mathrm{~L} \cdot \mathrm{mol}^{-1} \cdot \mathrm{cm}^{-1}$, 作者将该物质的强吸收特性归 因于苯环对于醌式结构的稳定作用. 化合物 8 的 $\mathrm{HOMO} / \mathrm{LUMO}$ 能级为 $-4.88 /-4.26 \mathrm{eV}$. 基于化合物 8 的场效应晶体管表现出双极性电荷传输特征 $\left(\mu_{\mathrm{e}}=8.9 \times\right.$ $\left.10^{-4} \mathrm{~cm}^{2} \cdot \mathrm{V}^{-1} \cdot \mathrm{s}^{-1}, \mu_{\mathrm{h}}=3.1 \times 10^{-2} \mathrm{~cm}^{2} \cdot \mathrm{V}^{-1} \cdot \mathrm{s}^{-1}\right)$.
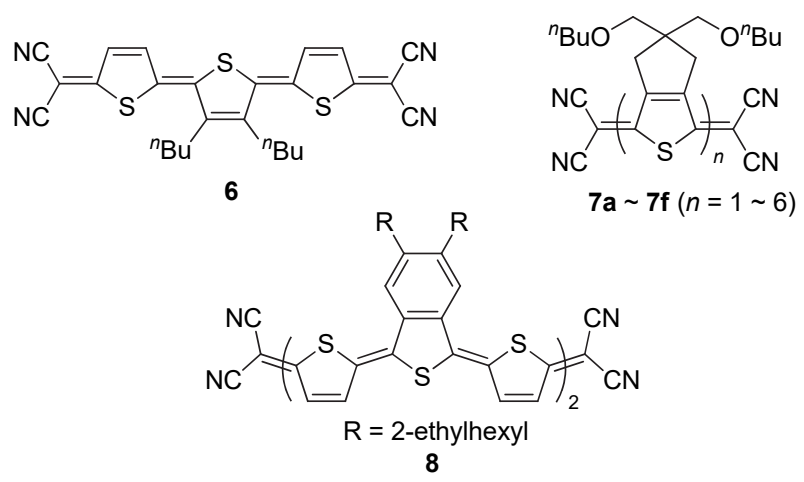

将醌式噻吩化合物中的噻吩单元替换为硒吩 ${ }^{[21,22] \text { 、 }}$ 吡咯 ${ }^{[23]}$ 、呋喃 ${ }^{[24]}$ 等杂环结构. 例如, 分子中引入硒吩通 常有助于增强分子间作用力. 2009 年, Takimiya 课题 组 ${ }^{[21]}$ 报道了含有硒吩单元的化合物 $\mathbf{9}$, 其 $\mathrm{HOMO} /$ LUMO 能级为 $-5.5 /-4.1 \mathrm{eV}$. 基于化合物 9 的场效应晶 体管表现出双极性电荷传输特征 $\mu_{\mathrm{e}}=1.6 \times 10^{-2} \mathrm{~cm}^{2}$ $\left.\mathrm{V}^{-1} \cdot \mathrm{s}^{-1}, \mu_{\mathrm{h}}=7.0 \times 10^{-3} \mathrm{~cm}^{2} \cdot \mathrm{V}^{-1} \cdot \mathrm{s}^{-1}\right)$. 分子中采用吡咯
结构则便于引入烷基链, 可以较好解决醌式杂环化合物 溶解性不佳的问题. 2012 年, 朱道本课题组 ${ }^{[23]}$ 报道了含 有吡咯结构的化合物 10, 单晶结构分析结果表明, 该化 合物分子仅含顺式结构. 其 LUMO 能级约为 $-4.30 \sim-$ $4.39 \mathrm{eV}, \mathrm{HOMO}$ 能级约为 $-5.48 \sim-5.56 \mathrm{eV}$. 基于化合 物 10a $\sim 10 \mathrm{c}$ 的场效应晶体管的电子迁移率最高为 0.014 $\mathrm{cm}^{2} \cdot \mathrm{V}^{-1} \cdot \mathrm{s}^{-1} .2016$ 年, 李洪祥课题组 ${ }^{[24]}$ 将醌式联三噻吩 中的中间噻吩单元替换为呋喃，设计合成了化合物 11, 其 HOMO/ LUMO 能级为 $-5.72 /-4.15 \mathrm{eV}$. 作者发现化 合物 11 具有很强的分子间 $\pi-\pi$ 及 $\mathrm{CN} \cdots \mathrm{H}$ (噻吩)相互作 用. 基于该化合物的有机场效应晶体管的电子迁移率最 高达到 $7.7 \mathrm{~cm}^{2} \cdot \mathrm{V}^{-1} \cdot \mathrm{s}^{-1}$.<smiles>N#CC(C#N)=c1ccc(=c2sc(=c3ccc(=C(C#N)C#N)[se]3)c3c2CC(CO)(CO)C3)[se]1</smiles>

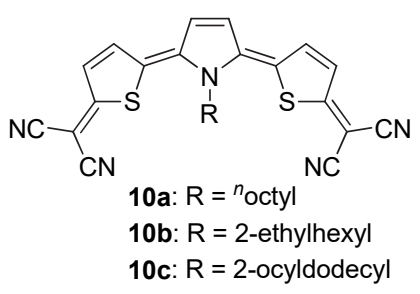

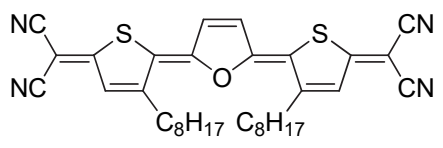

11

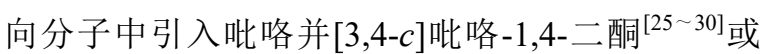
噻吩并 $[3,4-c]$ 吡咯-4,6-二䣯 ${ }^{[31 ~ 34]}$ 结构, 也是设计高性能 醌式杂环化合物的有效方法. 2012 年, 朱道本课题组 ${ }^{[25]}$ 报道了基于吡咯并 [3,4-c]吡咯-1,4-二酮结构的化合物 12, 其 LUMO 能级低至 $-4.51 \mathrm{eV} .2014$ 年, 朱道本课题 组 ${ }^{[27]}$ 进一步将噻吩并噻吩结构引入该体系中, 合成了 具有更大共轭体系的化合物 13, 其 LUMO 能级进一步 降低至 $-4.61 \mathrm{eV} .2019$ 年, 朱晓张课题组 ${ }^{[30]}$ 则设计合成 了同时含有噻吩并 $[3,4-b]$ 噻吩和吡咯并 [3,4-c]吡咯-1,4二酮单元的醌式化合物 14, 其 LUMO 能级为 $-4.37 \mathrm{eV}$. 基于化合物 12 14 的有机场效应晶体管的电子迁移率 分别达到 $0.55 、 0.22$ 和 $0.13 \mathrm{~cm}^{2} \cdot \mathrm{V}^{-1} \cdot \mathrm{s}^{-1}$. 在应用噻吩并 吡咯二酮结构方面, 2014 年, 朱晓张课题组 ${ }^{[31]}$ 采用噻吩 并 $[3,4-c]$ 吡咯-4,6-二酮和噻吩并 $[3,4-b]$ 噻吩结构作为醌 式结构单元, 设计合成了新型 $2 \mathrm{D}$ 醌式化合物 15 和 16. 分子间相互作用因共轭体系的增大而增大，而分子内 氧一硫和硫-硫等弱相互作用则增加了醌式结构的稳定 性. 其中, 基于化合物 15 的有机场效应晶体管的电子迁 移率达到 $3.0 \mathrm{~cm}^{2} \cdot \mathrm{V}^{-1} \cdot \mathrm{s}^{-1}$. 而对化合物 15 的烷基链进一 
步修饰后(化合物 17), 器件的电子迁移率提升至 5.2 $\mathrm{cm}^{2} \cdot \mathrm{V}^{-1} \cdot \mathrm{s}^{-1[32]}$.

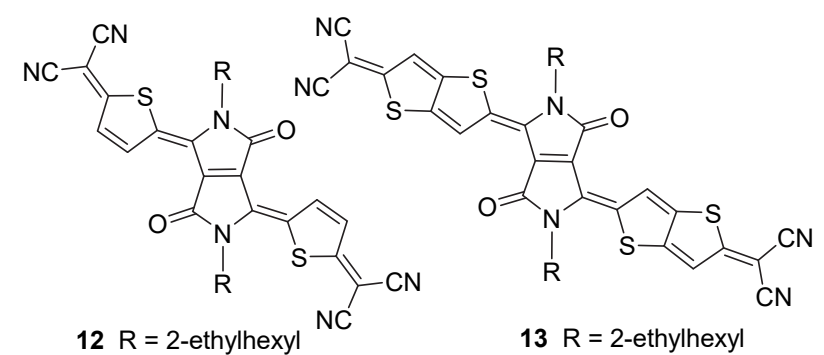

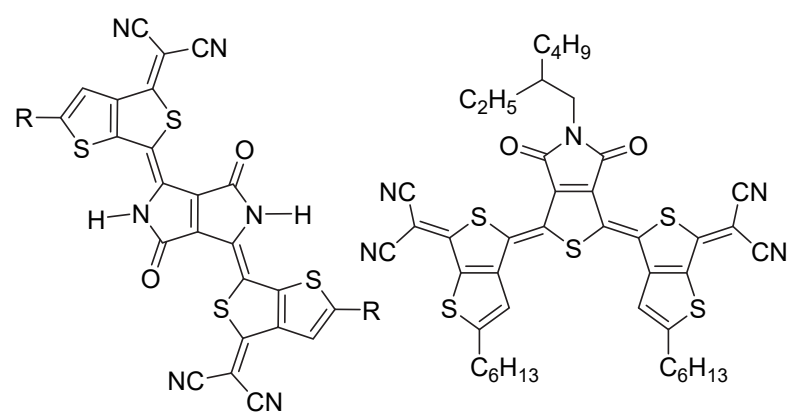

$14 \mathrm{R}=$ 2-hexyldecyl

15

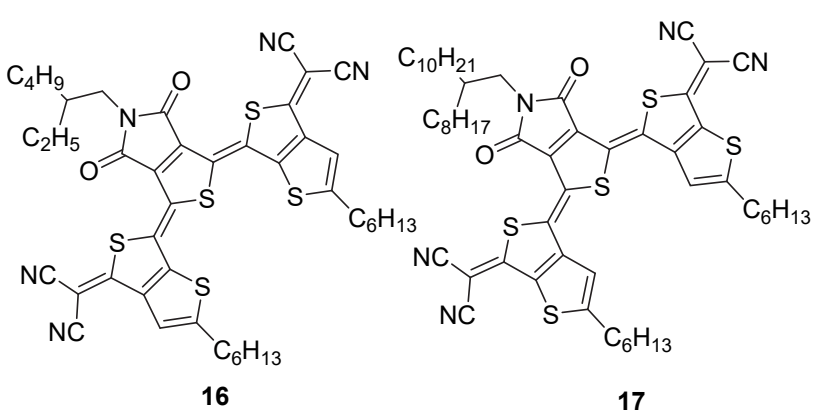

稠环类杂环母体的应用及其体系拓展. 由于稠环类 杂环母体的醌式化合物不存在顺反异构问题, 因此利于 对其进行结构表征、改善器件成膜效果和提高器件效率 等 $^{[35 \sim 43]} .2014$ 年, Takimiya 课题组 ${ }^{[39]}$ 以䒺并 $\left[1,2-b: 5,6-b^{\prime}\right]$ 二噻吩为母体, 设计合成了化合物 18 , 其 $\mathrm{HOMO} /$ LUMO 能级分别为 $-5.8 /-4.6 \mathrm{eV}$. 基于化合物 18 的有 机场效应晶体管的电子迁移率为 $0.1 \mathrm{~cm}^{2} \cdot \mathrm{V}^{-1} \cdot \mathrm{s}^{-1}$. 李荣 金和李洪祥等 ${ }^{[40]}$ 则以并四噻吩为母体, 设计合成了化 合物 19, 其 LUMO 能级为 $-4.3 \mathrm{eV}$, 基于该化合物的有 机场效应晶体管的电子迁移率达到 $0.9 \mathrm{~cm}^{2} \cdot \mathrm{V}^{-1} \cdot \mathrm{s}^{-1}$. 李 洪祥课题组 ${ }^{[41]}$ 进一步设计合成了以二噻吩并 $\left[2,3-d ; 2^{\prime}, 3^{\prime}-\right.$ $d^{\prime}$ 苯并 $\left[1,2-b ; 4,5-b^{\prime}\right]$ 二噻吩为母体的并五环醌式噻吩化 合物 20, 并发现虽然 20a、20b 和 20c 的 HOMO/LUMO 能级均为 $-5.68 /-4.39 \mathrm{eV}$, 但基于三者的有机场效应 晶体管却随着烷基链侧链位置的改变而呈现从单极性 电子传输向双极性电荷传输特征的转变趋势. Baumgarten 课题组 ${ }^{[42]}$ 以二噻吩并 $\left[2,3-d ; 2^{\prime}, 3^{\prime}-d\right]$ 苯并 $[2,1-b ; 3,4-$ $b^{\prime}$ 二噻吩为母体设计合成了化合物 $\mathbf{2 1}$, 并证明该化合 物以稳定的双自由基形式存在. 金铁男课题组 ${ }^{[43]}$ 则采
用拓展噻吩并吡咯体系的方法成功构建了具有并六环 及并七环结构的醌式化合物 22 和 $\mathbf{2 3}$, 其中化合物 23 的 $\mathrm{HOMO} / \mathrm{LUMO}$ 能级为 $-5.18 /-4.22 \mathrm{eV}$, 能级带隙仅为 $0.96 \mathrm{eV}$.

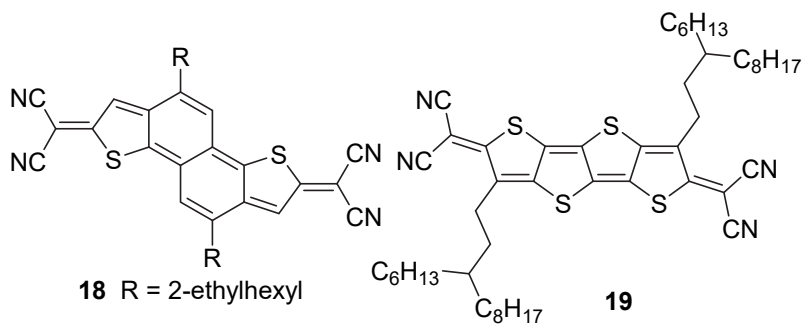

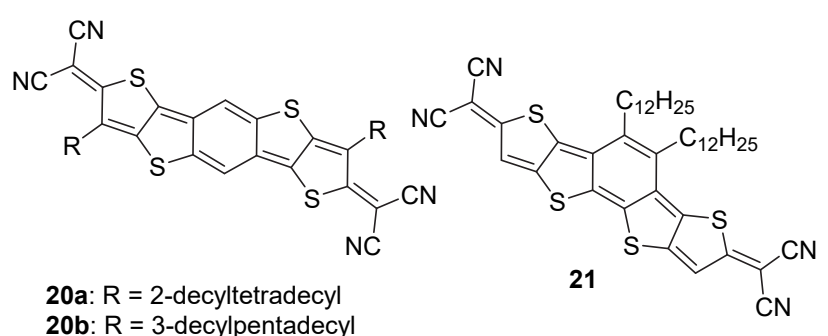
20b: $R=3$-decylpentadecyl 20c: $R=4$-decylhexadecyl

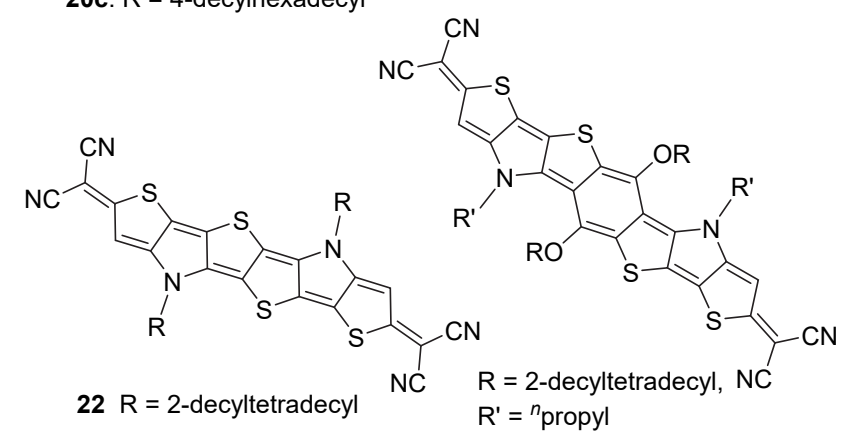

23

\section{2 (烷氧基)羰基氮基亚甲基醌式杂环化合物}

在二氧基亚甲基醌式噻吩分子体系中，随着醌式体 系的增大, 其溶解性会大幅下降. 为增加合成操作的便 捷性, 通常需要在醌式噻吩母体上引入很长的烷基链, 但这会影响分子的固体排列状态进而降低器件效率. 2010 年, Takimiya 课题组 ${ }^{[44]}$ 设计合成了一系列以(烷氧 基)羰基氰基亚甲基为端基的醌式噻吩化合物 $24 \sim 28$ (Scheme 3). 以(烷氧基)羰基替代氰基的思路具有以下 优点: (烷氧基)羰基仍具有一定的吸电性, 能够保证醌 式分子的稳定性; 便于进行烷基链修饰, 增加醌式分子 的溶解性; 烷基链沿醌式共轭长轴方向延伸, 有利于醌 式分子在成膜状态的有序排列. 作者采用了两种方法来 进行合成：第一种方法以氯甲基联噻吩为起始原料，分 步引入氰基和(烷氧基)羰基，再经 $N$-氯代丁二酰亚胺 (NCS) 氧化得到目标产物, 但总收率低于 $2 \%$ (Scheme 3). 第二种方法以溴代噻吩为原料, 经 Takahashi 反应和 氧化反应直接合成目标产物。该方法不仅合成步骤大幅 
缩减，总收率得到提升，且中间体更为稳定，易于实际 操作(Scheme 3). 因此, 作者进一步拓展了该体系的母 体结构. 聚四噻吩等塞噻吩及并三噻吩等稠环母体均适 用于该体系(化合物 $25 \sim 28$ ). 由于(烷氧基)羰基的吸电 性弱于氧基, 该系列醌式噻吩化合物的 LUMO 能级略 高于二氧基亚甲基醌式噻吩化合物, 但其 LUMO 值仍 略低于 $-4.0 \mathrm{eV}$, 符合成为在空气氛围中能够稳定存在 的 $\mathrm{n}$ 型半导体材料的基本要求. 基于该系列醌式化合物 的场效应晶体管的电子迁移率最高为 $0.015 \mathrm{~cm}^{2} \cdot \mathrm{V}^{-1}$. $\mathrm{s}^{-1}$.

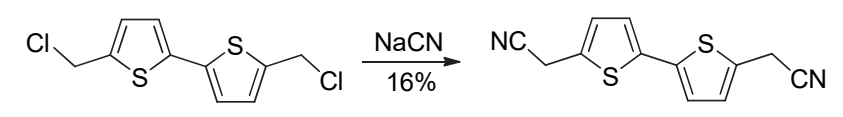<smiles>COC(=O)C(C)c1ccc(-c2ccc(C(C#N)C(C)OC(C)=O)s2)s1</smiles><smiles>COC(=O)/C(C#N)=c1/cc/c(=c2/cc/c(=C(\C#N)C(=O)OC)s2)s1</smiles><smiles>[R]OC(=O)/C(C#N)=c1\cc/c(=c2/cc/c(=C(/C#N)C(=O)O[R2])s2)s1</smiles>

图式 3 (烷氧基)羰基氰基亚甲基醌式联噻吩的合成路线 Scheme 3 Synthetic routes for (alkoxy)carbonylcyanomethylene quinoidal bithiophene<smiles>[R]OC(=O)C(C)=C1C=CC(=C(C)C(C)=O)SC1</smiles>

25a, 25b $(n=3,4)$

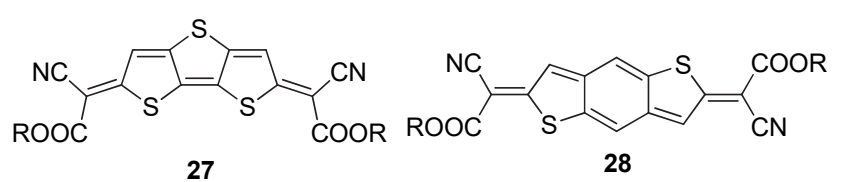

关于(烷氧基)羰基氰基亚甲基醌式噻吩衍生物的报 道较少 ${ }^{[45]} .2015$ 年, 朱晓张课题组 ${ }^{[46]}$ 在研究醌式噻吩化 合物的荧光性质时采用了(烷氧基)羰基氰基亚甲基作为 醌式化合物的端基. 由于醌式分子较易产生第一单重激 发态 $\left(\mathrm{S}_{1}\right.$ 态)到三重激发态 $\left(\mathrm{T}_{1}\right.$ 态)的隙间穿越, 醌式噻 吩化合物的苂光发射强度普遍极其微弱 ${ }^{[35]}$. 作者在(烷 氧基)羰基氰基亚甲基醌式联噻吩的母体结构上引入并 噻吩环和苯环, 合成了化合物 $29 \mathrm{a} \sim 29 \mathrm{~g}$, 成功将醌式化 合物的苂光发射能力 ${ }^{[47,48]}$ 加强了 $25 \sim 100$ 倍.

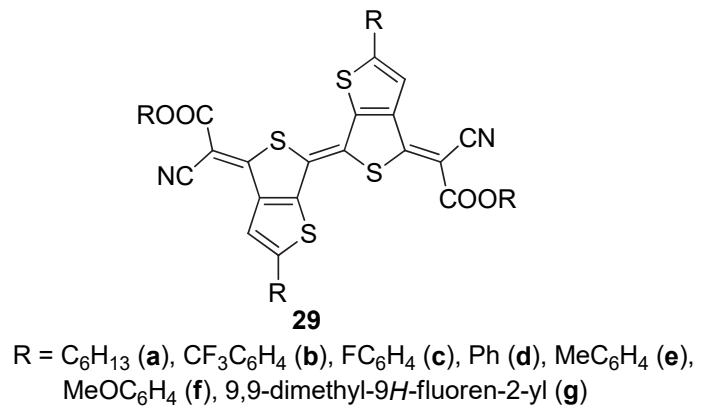

\section{3 酰基氨基亚甲基醌式杂环化合物}

受(烷氧基)羰基氰基亚甲基醌式噻吩衍生物的启 发, Takimiya 课题组 ${ }^{[49]}$ 还设想采用酰基氭基亚甲基作为 醌式杂环化合物的端基. 酰基氧基碳负离子因易于异构 形成烯醇式结构，使其反应活性受到限制，导致无法通 过 Takahashi 反应制备关键中间体 30 (Scheme 4). 因此, 作者采用分步合成策略向分子中引入氧基和酰基. 当先 引入氰基再接入酰基时, 酰基化易于过度反应而得到二 取代酰基化产物 31. 最后, 采用先引入酰基再接入㲵基 的策略, 成功得到了目标产物 32. 电化学结果表明, 酰

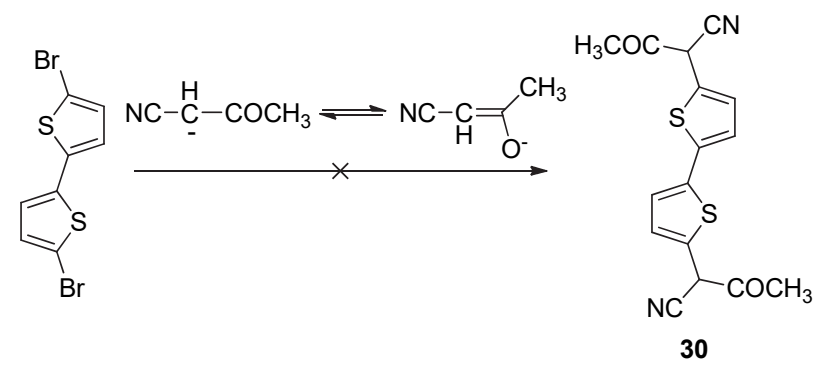<smiles>CC(=O)C(C)(C#N)c1ccc(-c2ccc(C(C#N)(C(C)=O)C(=O)OCc3ccc(CC#N)s3)s2)s1</smiles><smiles>[R]C(=O)Cc1cccs1</smiles><smiles>[R]C(=O)Cc1ccc(CC([R])C(=O)O[Ga])s1</smiles>

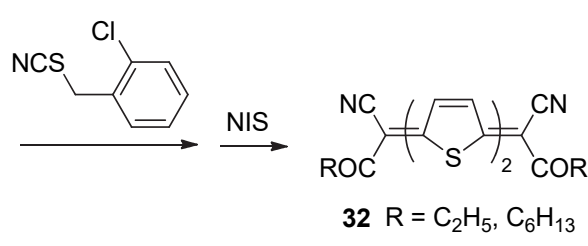

图式 4 酰基氰基亚甲基醌式噻吩衍生物的分子结构及其合 成路线

Scheme 4 Molecular structures and synthetic routes for acylcyanomethylene quinoidal thiophenes 
基的引入同样可以有效降低醌式化合物的 LUMO 能级 (约 $-4.2 \mathrm{eV}$ ), 使其成为有效的 $\mathrm{n}$ 型有机半导体材料. 经 测试, 基于该类物质的有机场效应晶体管的电子迁移率 最高为 $0.06 \mathrm{~cm}^{2} \cdot \mathrm{V}^{-1} \cdot \mathrm{s}^{-1}$. 由于合成路线较为复杂, 尚未 见该类醌式化合物的结构拓展及应用报道.

\section{4 二苯基亚甲基醌式杂环化合物}

二苯基亚甲基醌式杂环化合物的出现源自于对齐 齐巴宾化合物(Chichibabin hydrocarbon)的研究. 齐齐巴 宾存在双自由基形式与稳定醌式结构形式之间的互变 特性, 活泼的双自由基形态往往导致齐齐巴宾异常不稳 定. 为获得稳定的齐齐巴宾衍生物, 1991 年, Nakayama 课题组 ${ }^{[50]}$ 采用噻吩代替苯环设计合成了二苯基亚甲基 醌式噻吩化合物 33 (Scheme 5). 该合成路线首先合成二 苯基羟基甲基噻吩中间体，随后在高氯酸作用下脱去两 个羟基，再在金属锌的作用下得到醌式产物. 采用该方 法还可以得到醌式呋喃、醌式硒吩和醌式吡咯等衍生物, 也可以使用噻吩基代替苯基得到二噻吩基亚甲基醌式 噻吩化合物.

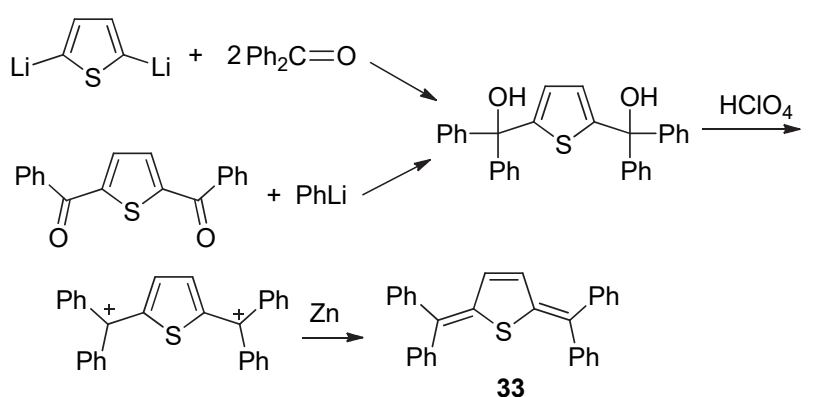

图式 5 二苯基亚甲基醌式噻吩的合成路线 Scheme 5 Synthetic route for diphenylmethylene quinoidal thiophene

然而, 上述合成路线中的正离子中间体的稳定性较 差, 以致不易分离. 为克服该缺点, Takeda 等 ${ }^{[51]}$ 在 2015 年对该合成路线进行了改进(Scheme 6). 在新路线中,
作者改用路易斯酸 $\mathrm{TMSClO}_{4}$ 来代替高氯酸进行脱羟基 反应，原位获得正离子中间体后再将 $\mathrm{TMSClO}_{4}$ 蒸除，加 入还原剂 $\mathrm{Zn}$ 得到产物. 采用该路线可将制备化合物 34 和 35 的最后一步反应收率从 $6 \%$ 大幅提升至 $99 \%$. 由于 给电子效应显著，二苯基亚甲基难以维持具有较大体系 的醌式噻吩结构, 该类醌式化合物的母体仅限于噻吩、 噻吩并噻吩和联噻吩 ${ }^{[52,53]}$.
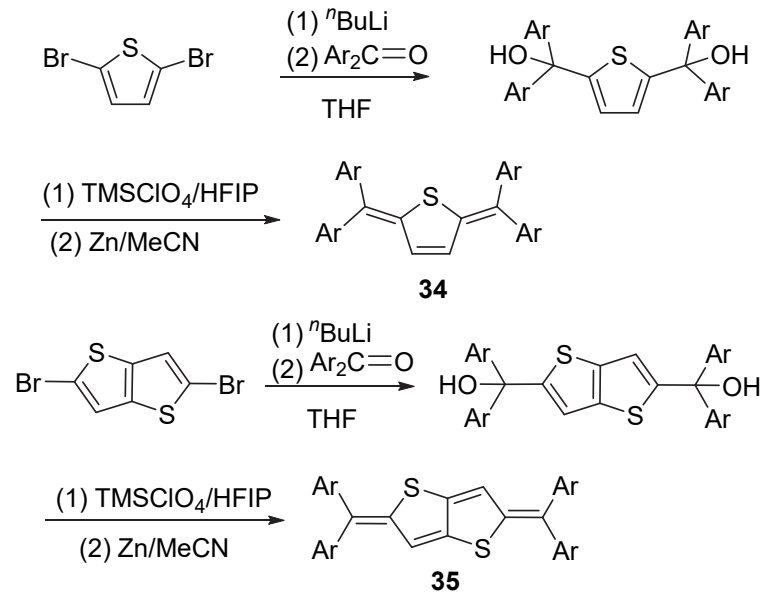

$\mathrm{Ar}=\mathrm{C}_{6} \mathrm{H}_{5}(\mathbf{a}), 4-\mathrm{MeOC}_{6} \mathrm{H}_{4}(\mathbf{b}), 4-\mathrm{ClC}_{6} \mathrm{H}_{4}(\mathbf{c})$

图式 6 改进后的二苯基亚甲基醌式噻吩的合成路线 Scheme 6 Modified synthetic route for diphenylmethylene quinoidal thiophenes

\section{5 亚芴基醌式杂环化合物}

与二苯基亚甲基醌式噻吩相比，亚芴基醌式噻吩具 有更高的结构稳定性. 已报道的衍生物包括亚芴基醌式 噻吩、亚芴基醌式噻吩并噻吩及亚芴基醌式联噻吩等. 1991 年, Kobayashi 等 ${ }^{[54]}$ 采用了多种方法来合成该类物 质. 以二(9-羟芴基)噻吩并噻吩(36)为原料合成目标化 合物 38 时, 既可利用氢碘酸在醋酸-醋酸酐体系中进行 回流脱除羟基得到中间体 37 , 再经脱氢氧化反应得到 目标产物，也可由原料直接经加热或光照得到(Scheme 7).

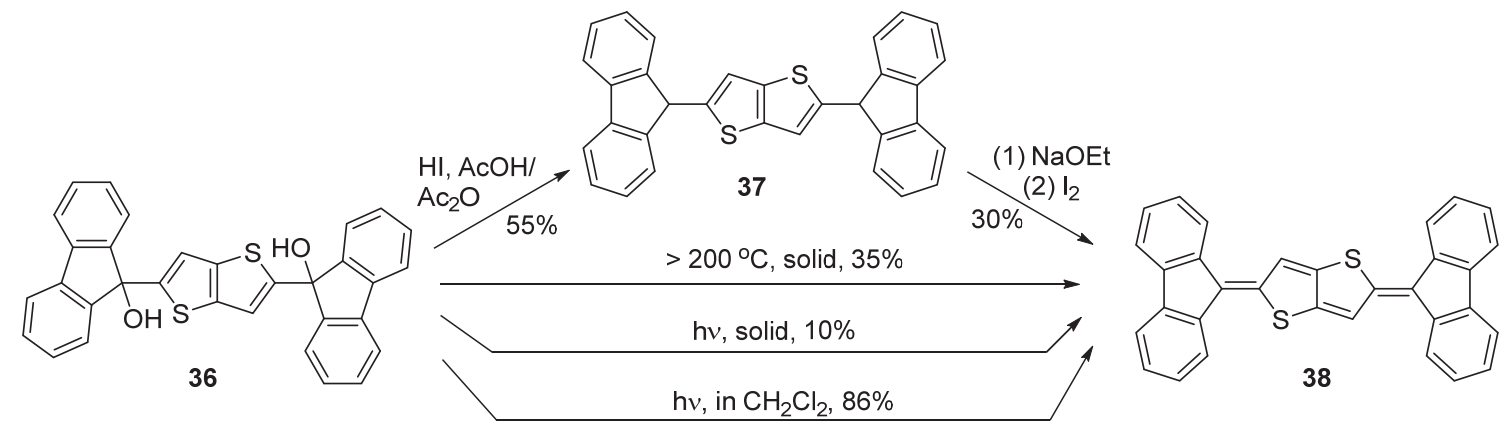

图式 72,5 -二(9-亚芴基)-2,5-醌式噻吩并 $[3,2-b]$ 噻吩的合成路线

Scheme 7 Synthetic routes for 2,5-bis(fluorene-9-ylidene)-2,5-dihydrothieno[3,2-b]thiophene 
单线态裂分材料是指物质受激发至第一单重激发 态 $\left(\mathrm{S}_{1}\right)$ 后, 裂分成两个三重激发态 $\left(\mathrm{T}_{1}\right)$ 载流子, 这样便可 以在理论上将光伏器件的外量子效率提高近两倍. 成为 单线态裂分材料需要在能量上满足 $E\left(\mathrm{~S}_{1}\right) \geqslant 2 E\left(\mathrm{~T}_{1}\right)$ 及在 空间上达到足够近的分子间距离. 研究表明, 部分醌式 杂环化合物能够满足成为单线态材料的要求. 2016 年, Kido 课题组 ${ }^{[55]}$ 在得到 2-(9-羟芴基)噻吩衍生物后采用氯 仿为溶剂、二氯化锡为脱着基试剂, 合成了亚芴基醌式 噻吩衍生物 38 40 (Scheme 8), 并将其作为电子给体材 料用于有机太阳能电池中, 虽然电池的光电转换效率只 有约 $1.1 \%$, 但却验证了二亚芴基醌式噻吩化合物作为 单线态材料在有机太阳能电池中应用的可行性.

\section{6 苯醌基醌式杂环化合物}

早在 1989 年, Takahashi 课题组 ${ }^{[56]}$ 就报道了以 2,6二叔丁基苯醌基为端基的醌式噻吩化合物. 以醌式
联噻吩化合物 46 为例, 合成路线如 Scheme 9 所示. 以 3,5-二叔丁基-4-三甲基硅氧基碘苯为起始原料, 采用 Negishi 反应和溴代反应制备中间体 42, 并制备锌试剂 43, 再将中间体 42 和 43 通过 Negishi 偶联反应合成中 间体 44, 随后使用盐酸脱甲硅基, 并采用铁氭化钾氧 化, 即得到目标产物 46. 采用类似的合成路线可以合成 具有 $1 \sim 4$ 个噻吩单元的二苯醌基醌式杂环化合物. 化 合物 46 的最大吸收波长为 $680 \mathrm{~nm}$ ，摩尔消光系数达到

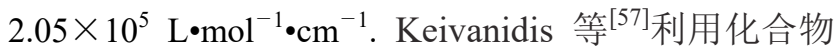
46 具有强烈吸收特征将其添加进 P3HT/PCBM 本体 异质结有机太阳能电池中, 将电池的光电转换效率 提升 $47 \%$.

与其它类型的醌式杂环化合物相似，苯醌基醌式杂 环化合物也存在着醌式结构形式与双自由基形式的平 衡关系. 双自由基形式会导致顺反异构问题加剧, 结构 不稳定性增加以及器件效率降低. 随着醌式噻吩单元的
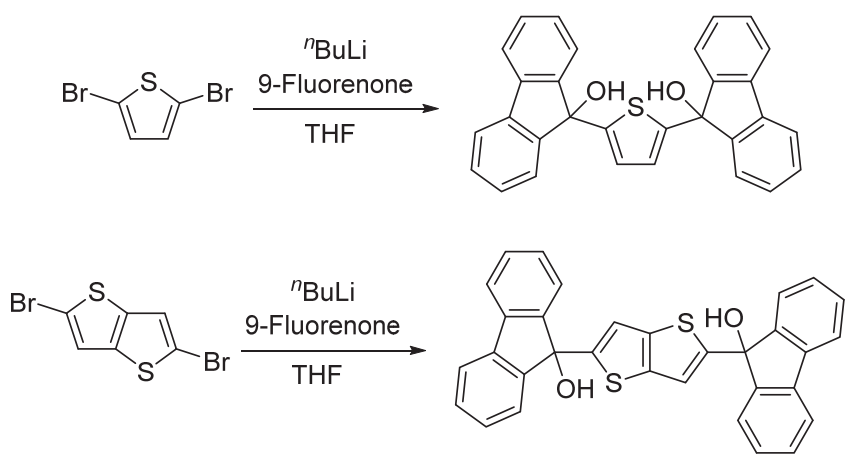

${ }^{n} \mathrm{BuL}$<smiles>Brc1ccc(-c2ccc(Br)s2)s1</smiles><smiles>OC1(c2ccc(-c3ccc(C4(O)c5ccccc5-c5ccccc54)s3)s2)c2ccccc2-c2ccccc21</smiles>

$\underset{\mathrm{CHCl}_{3}}{\stackrel{\mathrm{SnCl}_{2}}{\longrightarrow}}$
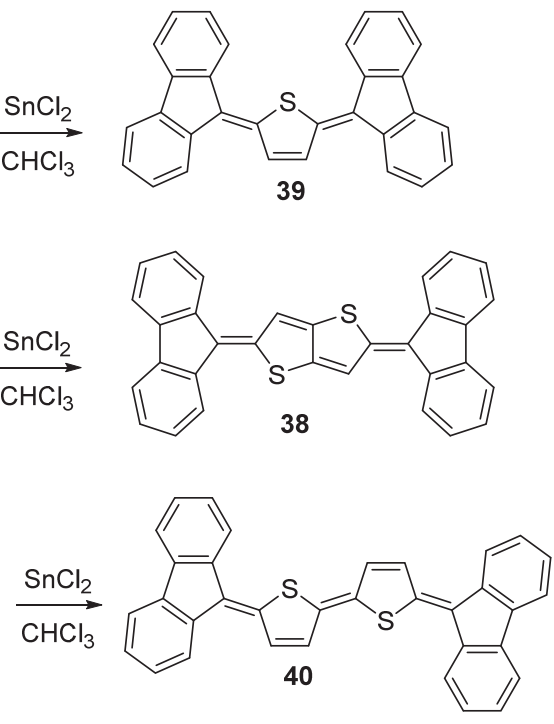

图式 8 醌式化合物 $\mathbf{3 8 \sim 4 0}$ 的分子结构及其合成路线

Scheme 8 Molecular structures and synthetic routes for quinoidal compounds $38 \sim \mathbf{4 0}$

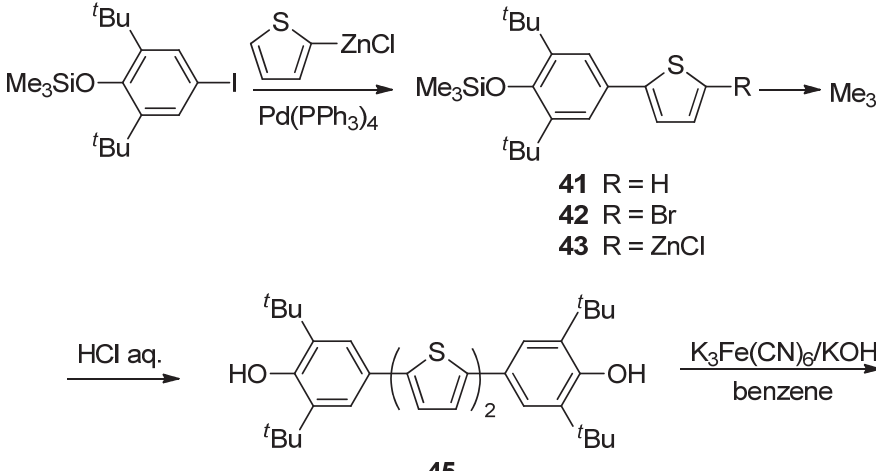

45<smiles>COc1c(C(C)(C)C)cc(-c2ccc(-c3cc(C(C)(C)C)c(O)c(C(C)(C)C)c3)s2)cc1C(C)(C)C</smiles>

44<smiles>CC(C)(C)C1=CC(=c2ccc(=C3C=C(C(C)(C)C)C(=O)C(C(C)(C)C)=C3)s2)C=C(C(C)(C)C)C1=O</smiles>

46

图式 9 二苯醌基醌式噻吩化合物 46 的分子结构及其合成路线

Scheme 9 Molecular structure and synthetic route for $p$-diphenoquinone 46 
增加，双自由基形式所占比例越来越大，极大地限制了 苯醌基醌式杂环化合物在器件中的应用 ${ }^{[58,59]}$. Bertarelli 课题组 ${ }^{[60 ~ 62]}$ 设想通过在固定长度苯醌基醌式噻吩结构 上调节醌式母体上的取代基来调节醌式结构形式与双 自由基形式的平衡. 他们设计并合成了醌式化合物 47 54. 结果表明, 在该类醌式联噻吩结构的噻吩单元 上引入供电子基团或在端基苯醌结构上引入吸电子基 才, 有利于增强醌式结构的稳定性, 而在端基苯醌单元 上引入供电子基团更易诱发产生双自由基.<smiles>[R]C1=CC(=c2cc([R])c(=c3sc([R])cc3=C3C=C([R1])C(=O)C([R])=C3)s2)C=C([R])C1=O</smiles>

$47 \mathrm{R}^{1}={ }^{t} \mathrm{Bu}, \mathrm{R}^{2}=\mathrm{OMe}, \mathrm{R}^{3}=\mathrm{H}$

$48 \mathrm{R}^{1}={ }^{t} \mathrm{Bu}, \mathrm{R}^{2}=\mathrm{R}^{3}=\mathrm{OC}_{5} \mathrm{H}_{11}$

$49 \mathrm{R}^{1}={ }^{i} \mathrm{Pr}, \mathrm{R}^{2}=\mathrm{R}^{3}=\mathrm{OC}_{5} \mathrm{H}_{11}$

$50 \mathrm{R}^{1}=\mathrm{Me}, \mathrm{R}^{2}=\mathrm{R}^{3}=\mathrm{OC}_{5} \mathrm{H}_{11}$

$51 \mathrm{R}^{1}=\mathrm{H}, \mathrm{R}^{2}=\mathrm{R}^{3}=\mathrm{OC}_{5} \mathrm{H}_{11}$

$52 \mathrm{R}^{1}={ }^{\mathrm{t}} \mathrm{Bu}, \mathrm{R}^{2}=\mathrm{R}^{3}=\mathrm{COOH}$

$53 \mathrm{R}^{1}={ }^{t} \mathrm{Bu}, \mathrm{R}^{2}=\mathrm{R}^{3}=\mathrm{CH}\left(\mathrm{OCH}_{2}\right)_{2}$

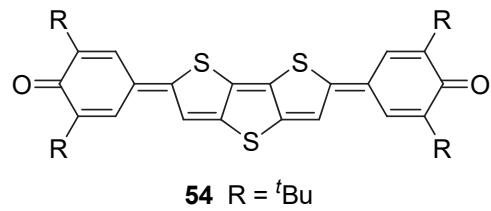

\section{7 靛吩咛衍生物}

靛吩咛是指以 2-吲哚酮为端基的醌式联噻吩化合 物. 以靛红和噻吩为原料, 在浓硫酸作用下生成蓝色靛 吩咛的反应, 被称之为 “靛吩咛反应” 。早期, 该反应被 用于鉴别噻吩, 但人们并不清楚所得蓝色物质的精确结 构. 直到 1993 年, Cava 等 ${ }^{[63]}$ 使用 $N$-庚烷基靛红为原料
合成了易溶于有机溶剂且能分离的 $N, N^{\prime}$-二庚烷基靛吩 咛衍生物 55 , 才确认靛吩咛为含有六个顺反异构形式 的醌式联噻吩化合物(Eq. 2).

2015 年, Kim 课题组 ${ }^{[64]}$ 采用靛吩咛反应制备了 $N, N^{\prime}$-二(十二烷基)靛吩咛(56)及其硒吩衍生物 $\mathbf{5 7}$, 并将 其作为半导体材料应用于有机场效应晶体管，发现该类 物质具有空穴流动性和电子流动性的双极性传输特征. 与二氰基亚甲基醌式化合物相比，靛吩咛的 HOMO/ LUMO 能级较高. 化合物 $\mathbf{5 6}$ 的 HOMO/LUMO 能级为 $-5.25 /-3.76 \mathrm{eV}$, 化合物 57 的 HOMO/LUMO 能级为 $-5.33 /-3.73 \mathrm{eV}$. 较高的 HOMO 能级可能是靛吩咛能 够产生空穴流动特征的原因.

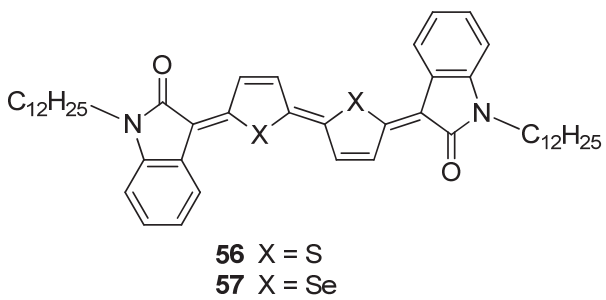

为开发基于靛吩咛结构的 $\mathrm{p}$ 型半导体材料, 朱晓张 课题组 ${ }^{[65]}$ 采用噻吩并 $[3,4-b]$ 噻吩结构替代靛吩咛中的 噻吩结构, 设计了二噻吩并靛吩咛衍生物 58 和 59. 为 保证分子中噻吩并噻吩结构的取向，作者采用了如 Scheme 10 所示的合成路线来合成化合物 58 和 59. 核磁 共振波谱数据表明该类分子结构并不存在异构现象. 化 合物 58 的 HOMO/LUMO 能级为 $-5.10 /-3.58 \mathrm{eV}$, 而化 合物 59 的 HOMO/LUMO 能级为 $-5.13 /-3.49 \mathrm{eV}$, 基于 化合物 58 和 59 的有机场效应晶体管的空穴迁移率分别 为 0.22 和 $0.055 \mathrm{~cm}^{2} \cdot \mathrm{V}^{-1} \cdot \mathrm{s}^{-1}$. 基于此，作者还将化合物 58 和 59 作为电子给体材料用于有机太阳能电池中, 分 别取得 $3.41 \%$ 和 $4.07 \%$ 的光电转换效率.

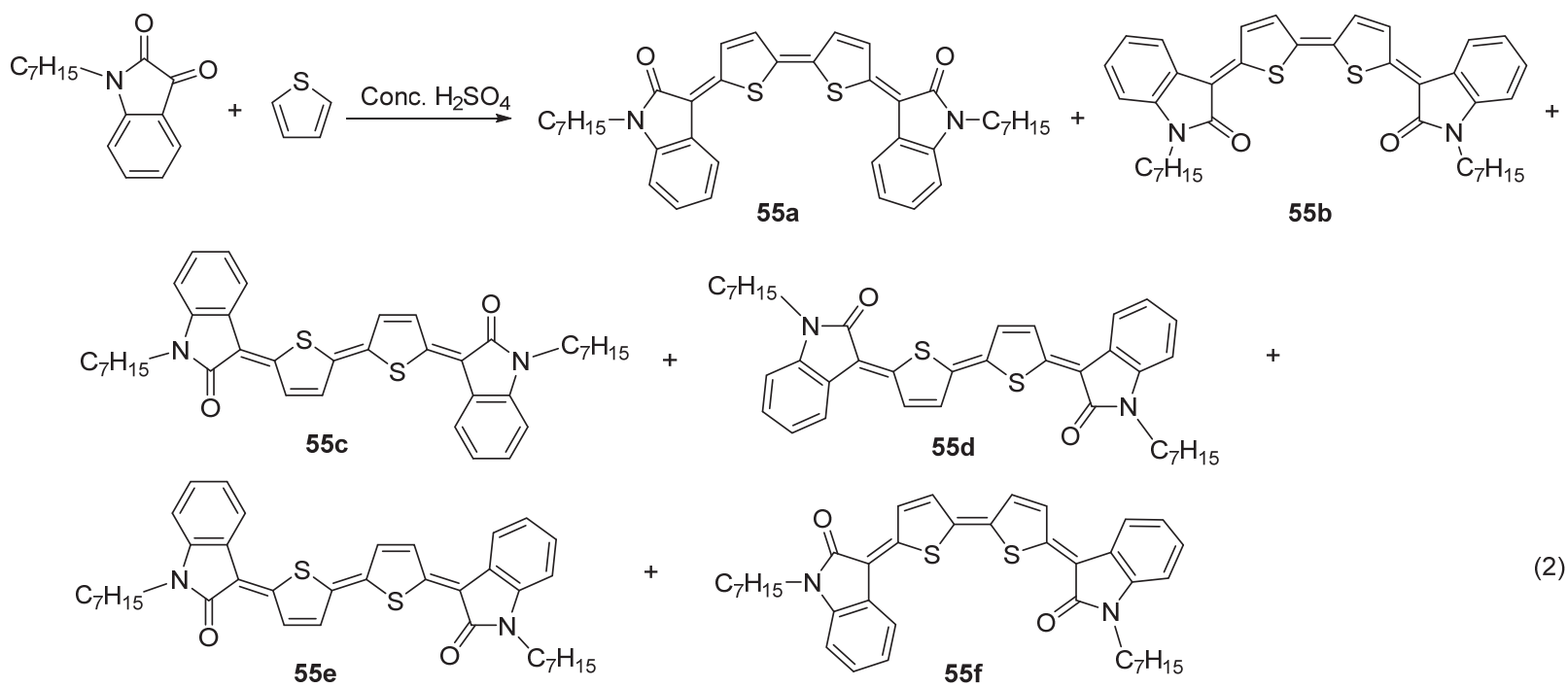




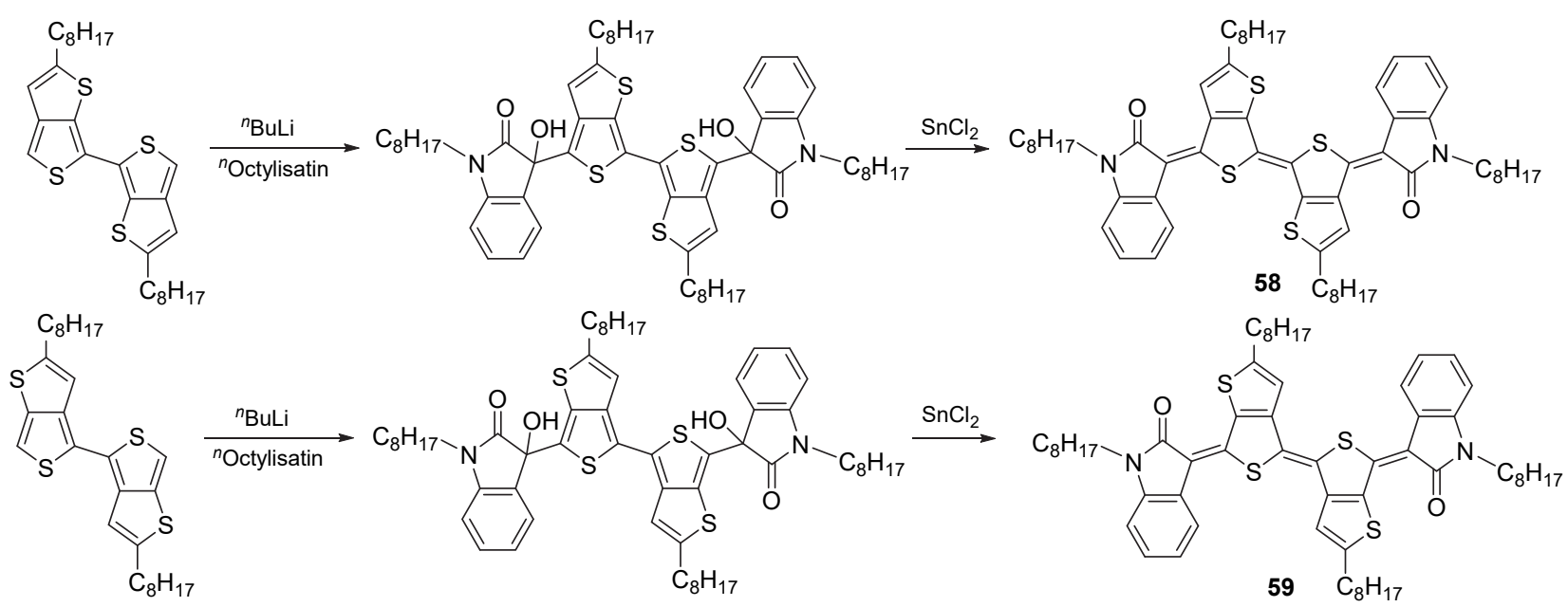

图式 10 噻吩并噻吩型靛吩咛衍生物的分子结构及合成路线

Scheme 10 Molecular structures and synthetic route of indophenine derivatives based on thienothiophene moiety

为实现静吩咛结构的单极性电子流动, 李玉宁课题 组 ${ }^{[66]}$ 采用间氯过氧苯甲酸将靛吩咛衍生物氧化, 得到 化合物 60a 60c. 氧化过程使得靛吩咛的六种异构形 式全部转变为反式结构, 从而方便了结构表征以及促进 固体状态的分子有序排列. 经过氧化的三只靛吩咛衍生 物 60a $\sim 60 \mathrm{c}$ 的 HOMO/LUMO 能级分别下降至 -5.85 / $-3.99 、-6.33 /-4.12$ 和 $-6.30 /-4.18 \mathrm{eV}$. 最终该类物 质在有机场效应晶体管中的电子迁移率最高为 0.11 $\mathrm{cm}^{2} \cdot \mathrm{V}^{-1} \cdot \mathrm{s}^{-1}$.

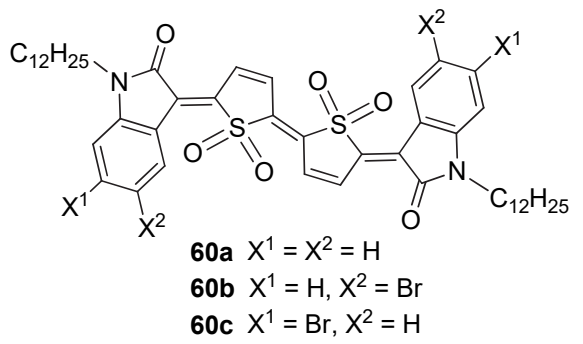

李玉宁课题组 ${ }^{[67,68]}$ 进一步将氧化靛吩咛结构作为 共聚单元制备成 “给体-受体” 型聚合物, 并将其作为半 导体材料应用在有机场效应晶体管中. 基于化合物 61

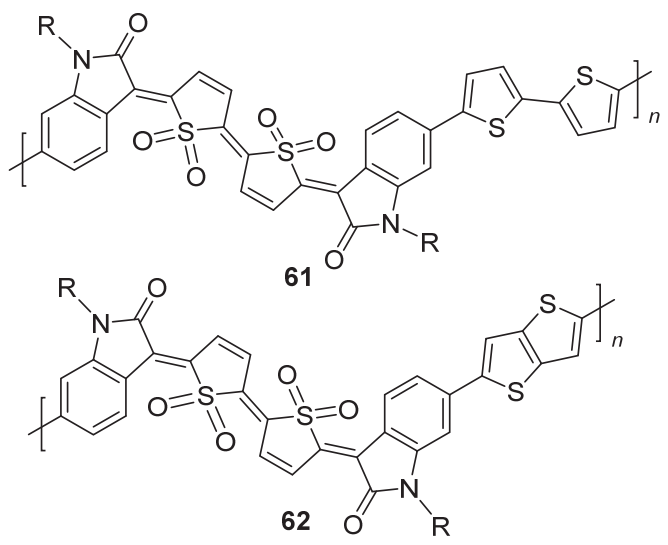

和 62 的有机场效应晶体管的电子流动率分别为 0.18 和 $0.14 \mathrm{~cm}^{2} \cdot \mathrm{V}^{-1} \cdot \mathrm{s}^{-1}$.

\section{8 以 DACP 为端基的醌式杂环化合物}

2005 年, Okada 课题组 ${ }^{[69]}$ 设计合成了以 $1,4-$ 二氮杂 环戊二烯(1,4-Diazacyclopentadienylidene, DACP)为端基 的醌式杂环化合物 $63 \sim 65$ (Scheme 11). 以化合物 63 为 例, 其合成路线以 2,5-二甲酰基噻吩为原料, 在醋酸铵醋酸介质中与偶苯酰进行缩合反应，得到相应中间体 后, 采用铁氧化钾进行氧化, 得到最终的醌式杂环化合 物. 化合物 $63 \sim 65$ 由于醌式 $\pi$ 共轭体系较小, 因而具有 较好的结构稳定性.

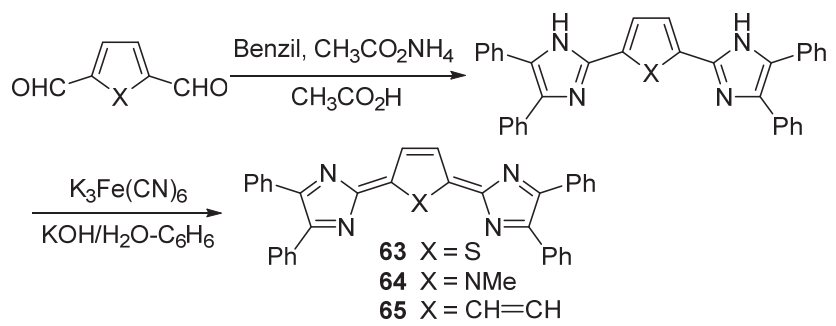

图式 11 DACP 型醌式噻吩化合物 $\mathbf{6 3} \sim \mathbf{6 5}$ 的分子结构及其合 成路线

Scheme 11 Molecular structures and synthetic routes for DACP type quinoidal thiophene compounds $63 \sim 65$

2006 年, Okada 课题组 ${ }^{[70]}$ 又报道了以醌式联噻吩为 母体的 DACP 型醌式杂环化合物 66. 作者采用了与化合 物 63 相同的合成路线来制备化合物 66 . 但与 63 相比, 化合物 66 已经表现出明显的双自由基特征.<smiles>c1ccc(-c2nc(-c3ccc(-c4ccc(-c5nc(-c6ccccc6)c(-c6ccccc6)[nH]5)s4)s3)c(-c3ccccc3)[nH]2)cc1</smiles> 
Nakamura 课题组 ${ }^{[71]}$ 鉴于醌式杂环化合物良好的吸 收特性，尝试将以 DACP 为端基的醌式单噻吩衍生物用 作电子给体材料, 应用于有机太阳能电池中. 为提升给 电子能力, 在 DACP 型醌式杂环分子上引入甲氧基, 设 计合成了化合物 $67 \sim 69$. 虽然以该类物质制作的有机 太阳能电池的光电转换效率还有待提升(仅有 $0.3 \%$ ), 但 其光电响应范围能够拓展至近红外区域(约 $890 \mathrm{~nm}$ ), 为 利用近红外区域的光能提供了有益的思路.

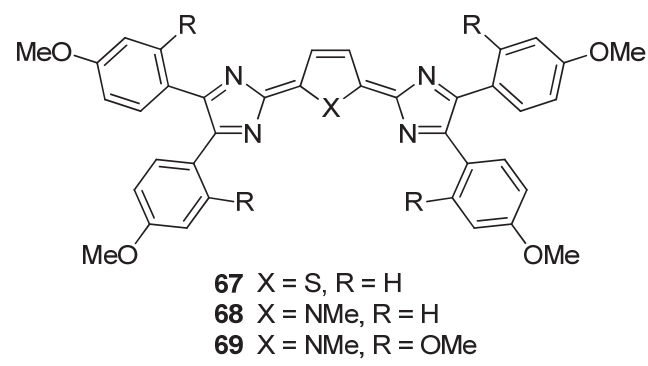

\section{9 多环芳烃型醌式杂环化合物}

采用多环芳烃的结构设计方式也是构造醌式杂环 分子的方法之一. 大共轭体系的多环芳烃通常因具有较 高的 HOMO 能级而使得结构稳定性不足. 采用醌式杂 环结构作为多环芳烃的内核可以有效降低 HOMO 能级 而增加结构稳定性. 2014 年, Haley 等 ${ }^{[72]}$ 设计合成了狮并 醌式噻吩衍生物 70 72, 其 HOMO 能级分别为 -5.77 、 -5.66 和 $-5.63 \mathrm{eV}, \mathrm{LUMO}$ 能级分别为 $-3.84 、-3.97$ 和 $-4.03 \mathrm{eV}$. Chi 课题组 ${ }^{[73]}$ 研究表明, 随着醌式共轭体 系的增大，化合物 73 已含有一定量的双自由基形式，使 其表现出较高的活泼性.

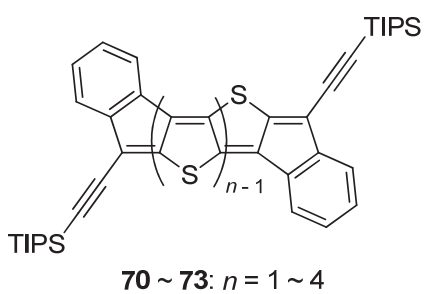

2015 年, Chi 课题组 ${ }^{[74]}$ 又报道了醌式噻吩化合物 74, 在二氯甲烷中的最大吸收波长为 $682 \mathrm{~nm}\left(\varepsilon=1.07 \times 10^{5}\right.$ $\left.\mathrm{L} \cdot \mathrm{mol}^{-1} \cdot \mathrm{cm}^{-1}\right), \mathrm{HOMO} / \mathrm{LUMO}$ 能级为 $-4.65 /-3.46$

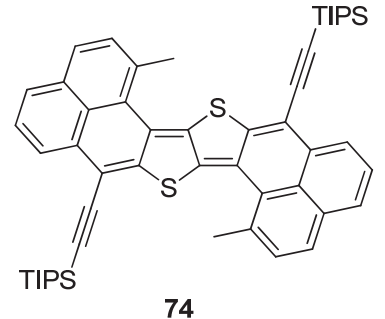

$\mathrm{eV}$. 基于化合物 74 的有机场效应晶体管的空穴迁移率 为 $0.26 \mathrm{~cm}^{2} \cdot \mathrm{V}^{-1} \cdot \mathrm{s}^{-1}$.

2016 年, Chi 课题组 ${ }^{[75]}$ 进一步设计合成了醌式化合 物 75 78, 并研究了该类物质的基态结构特征. $75 \sim 78$ 的最大吸收波长分别为 570、640、666 和 $702 \mathrm{~nm}, \mathrm{HOMO}$ 能级范围为 $-4.39 \sim-4.77 \mathrm{eV}, \mathrm{LUMO}$ 能级范围为 $-3.06 \sim-3.40 \mathrm{eV}$. 随着醌式体系的增大，基态时的双 自由基特征指数则由化合物 75 的 0 增大至化合物 78 的 $38.2 \%$.

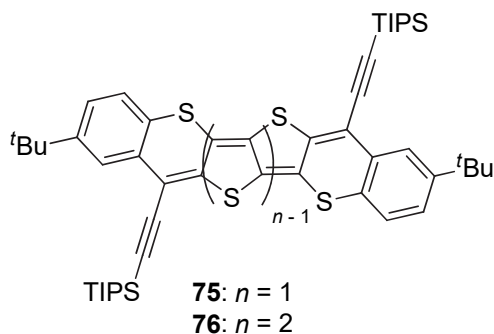

$77: n=3$

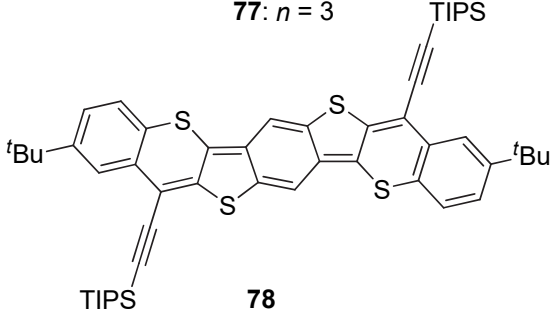

\section{0 “D-A”型醌式杂环化合物}

通过构建 “电子给体-电子受体” 型(即 “D-A” 型) 分子，可以引入分子内电荷转移特征，从而拓展分子的 吸收光谱, 增强吸光能力. 具有推拉电子体系的 “D-A” 型分子在有机太阳能电池、非线性光学材料及近红外染 料领域均有广泛应用 ${ }^{[76]}$. 在醌式结构中, 若分别采用给 电子基团和吸电子基团作为端基，即可形成典型的 “D-A” 分子.

1997 年, Otsubo 课题组 ${ }^{[77]}$ 设计了以 1,3-二硫杂环戊 烯和二氰基亚甲基为醌式杂环结构端基的 “ $\mathrm{D}-\mathrm{A}$ ” 型醌 式杂环化合物 79 81. 首先采用 Takahashi 反应将二氰 基甲基连接到噻吩单元上，再与 2-甲硫基-1,3-二硫杂环 戊烯硫酸甲酯缩合即得到化合物 79a. 采用该方法还可 以合成出硒吩、碲吩、呋喃衍生物以及联噻吩、噻吩并 $[3,2-b]$ 噻吩等衍生物. 研究发现, 醌式单杂环衍生物 79 的最大吸收波长为 $533 \sim 561 \mathrm{~nm}$, 醌式联噻吩(硒吩)衍 生物 80 的最大吸收波长为 $693 \sim 739 \mathrm{~nm}$ ，醌式噻吩并噻 吩衍生物 81 的最大吸收波长为 $622 \sim 625 \mathrm{~nm} .2017$ 年, Holzmüller 等 ${ }^{[78]}$ 以化合物 $\mathbf{8 0 a}$ 为给体材料、以 $\mathrm{C}_{60}$ 为受 体材料制作了体相异质结有机太阳能电池，取得 $1.9 \%$ 的光电转换效率. 
<smiles>[R]C1=CC(=C2SC([R])=C([R])S2)C(=[X])C1=C(C)N</smiles>

79a: $X=S, R=R^{\prime}=H$

79b: $X=S, R={ }^{n} \mathrm{C}_{8} \mathrm{H}_{17} \mathrm{~S}, \mathrm{R}^{\prime}=\mathrm{H}$

79c: $X=S, R=H, R^{\prime}={ }^{n} \mathrm{C}_{6} \mathrm{H}_{13}$

79d: $X=S, R={ }^{n} \mathrm{C}_{8} \mathrm{H}_{17} \mathrm{~S}, \mathrm{R}^{\prime}={ }^{n} \mathrm{C}_{6} \mathrm{H}_{13}$

79e: $X=\mathrm{Se}, \mathrm{R}=\mathrm{R}^{\prime}=\mathrm{H}$

79f: $X=\mathrm{Se}, \mathrm{R}={ }^{n} \mathrm{C}_{8} \mathrm{H}_{17} \mathrm{~S}, \mathrm{R}^{\prime}=\mathrm{H}$

79g: $X=T e, R=R^{\prime}=H$

79h: $X=T e, R={ }^{n} \mathrm{C}_{8} \mathrm{H}_{17} \mathrm{~S}, \mathrm{R}^{\prime}=\mathrm{H}$

79i: $X=O, R=R^{\prime}=H$

79j: $X=O, R={ }^{n} \mathrm{C}_{8} \mathrm{H}_{17} \mathrm{~S}, \mathrm{R}^{\prime}=\mathrm{H}$

布于整个分子的共轭体系上，而 LUMO 状态的电子云 则主要位于醌式噻吩及氧基上. 以化合物 85 作为光敏 染料制作的 $\mathrm{p}$ 型染料敏化太阳能电池的光电转换效率为 $0.5 \%$, 其中, 短路电流密度达到 $8.2 \mathrm{~mA} \cdot \mathrm{cm}^{-2}$.

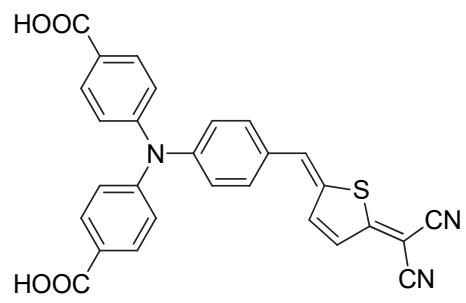

85

为增加 “D-A” 型醌式杂环化合物的溶解性, 2005 年, Otsubo 课题组 ${ }^{[79]}$ 进一步设计合成了以 5,5-双(丁氧基 甲基)-5,6-二氢- $4 H$-环戊烷并 $[c]$ 噻吩为醌式 $\pi$ 桥的醌式 噻吩化合物 82 和 83 . 与化合物 $79 \mathrm{a}\left(2.3 \times 10^{-3} \mathrm{~mol} / \mathrm{L}\right)$ 和 $80 \mathrm{a}\left(<10^{-5} \mathrm{~mol} / \mathrm{L}\right)$ 相比, 82 和 83 在氯仿中的溶解度 $(>$ $10^{-2} \mathrm{~mol} / \mathrm{L}$ ) 有了大幅提升. 化合物 82 和 83 的吸收波长 随着溶剂极性的增加而红移, 表明该电子推拉体系具有 良好的分子内电荷转移特征.

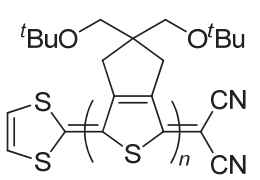

82a: $n=1$ 82b: $n=2$

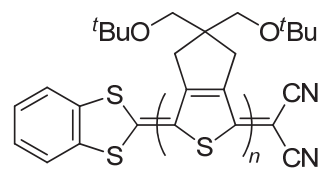

83a: $n=1$

83b: $n=2$

83c: $n=3$

2009 年, Liu 等 ${ }^{[80}$ 设计合成了醌式化合物 84. 他们 采用缩合反应将二氰基亚甲基醌式联噻吩与四硫富瓦 烯连接起来, 使二者成为一个完整的共轭体系. 通过理 论计算发现, HOMO 状态的电子云主要分布于四硫富瓦 烯结构上, 而 LUMO 状态的电子云则位于醌式噻吩母 体上, 这表明化合物 84 也是典型的具有电子推拉效应 的 “D-A” 型分子.

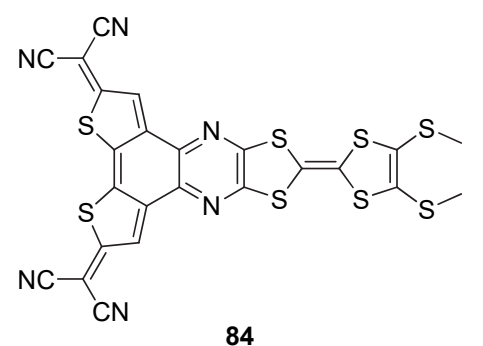

2015 年, Jiang 等 ${ }^{[81]}$ 报道了以三苯胺为给体、二氧基 亚甲基为受体、醌式噻吩为 $\pi$ 桥的化合物 85 , 最大吸收 波长为 $550 \mathrm{~nm}$, 摩尔消光系数为 $5.8 \times 10^{4}{\mathrm{~L} \cdot \mathrm{mol}^{-1}}^{-1}$ $\mathrm{cm}^{-1}$. 量子化学计算结果发现, HOMO 状态的电子云分

2019 年, Gräßler 等 ${ }^{[82]}$ 进一步设计合成了分别以苯 并噻唑与二氰基亚甲基为端基的醌式噻吩化合物 86 以 及吡喃与二氰基亚甲基为端基的醌式噻吩化合物 87 . 化合物 86 和 87 在二甲基亚砜(DMSO)中的最大吸收波

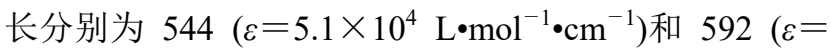
$\left.3.0 \times 10^{4} \mathrm{~L} \cdot \mathrm{mol}^{-1} \cdot \mathrm{cm}^{-1}\right) \mathrm{nm}, \mathrm{HOMO} / \mathrm{LUMO}$ 能级分别为 $-4.94 /-3.20 \mathrm{eV}$ 和 $-5.08 /-3.54 \mathrm{eV}$.

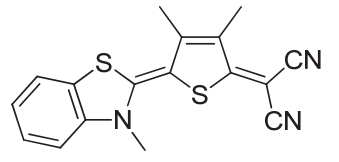

86<smiles></smiles>

87

\section{1 总结与展望}

综述了各类型醌式杂环化合物的分子结构特点、设 计合成方法及其应用性能. 理解醌式杂环分子结构与光 物理、电化学及应用性能之间的构效关系是进行醌式杂 环分子设计的必要基础. 从已报道的文献中可总结如 下: (1)醌式分子平面刚性的结构特点有利于缩小分子间 距离, 提升分子间电荷传输性能, 但却可能会大幅降低 溶解性; 合理引入烷基链可以有效增加溶解性, 但却可 能改变分子间排列方式. (2)醌式室噻吩衍生物通常包含 多种顺反异构体，不利于进行结构表征及制膜应用等; 消除异构现象的手段包括引入分子内 $\mathrm{S} \cdots \mathrm{S}$ 或 $\mathrm{S} \cdots \mathrm{O}$ 等 弱作用, 采用吡咯或呋喃等特殊结构单元, 以及采用稠 杂环母体等. (3) HOMO/LUMO 能级是醌式杂环化合物 作为半导体材料的重要参数. 醌式杂环分子通常具有较 低的 $\mathrm{HOMO} / \mathrm{LUMO}$ 能级, 其中, 绝大部分以氰基为端 基的醌式分子的 LUMO 能级小于 $-4.0 \mathrm{eV}$, 符合成为在 空气氛围中稳定的 $\mathrm{n}$ 型半导体材料的要求. (4)醌式杂环 分子具有很窄的能级带隙, 且随着醌式共轭体系的增大 而大幅缩小. 这也是醌式杂环化合物在可见光区及近红 外光区具有强吸收的重要原因, 使其具有作为光电材料 
或近红外材料的潜质. (5)本综述所列分子内端基相同的 醌式化合物的 HOMO/LUMO 电子云均匀分布于整个醌 式共轭体系上, 为引入分子内电荷传输特性, 设计 “D-A” 型醌式分子是有效方法. 常见设计思路包括在醌 式结构两端分别同时使用供电性端基和吸电性端基, 以 及将整个醌式结构作为受体单元与富电性结构缩合. (6) 醌式杂环化合物还是为数不多的可以制备稳定双自由 基的物质来源，同时作为单线态裂分材料也受到众多研 究者的关注. 醌式杂环化合物的发展仍然处于起步阶 段. 当前, 醌式杂环化合物已在有机场效应晶体管、有 机太阳能电池、非线性光学材料、近红外材料和纺织染 料 ${ }^{[83,84]}$ 等领域受到广泛关注. 未来, 新结构的设计开 发、性能研究及应用探索仍将是醌式杂环化合物的研究 方向.

\section{References}

[1] Casado, J.; Rocio, P. O.; Juan, T. N. Chem. Soc. Rev. 2012, 41, 5672.

[2] Burrezo, P. M.; Zafra, J. L.; Navarrete, J. T. L.; Casado, J. Angew. Chem., Int. Ed. 2017, 56, 2250.

[3] Wang, R.-H.; Qiao, X.-L.; Li, H.-X. Chin. Sci. Bull. 2016, 61, 296 (in Chinese).

(王瑞豪，乔小兰，李洪祥，科学通报, 2016, 61, 296.)

[4] Horowitz, G.; Kouki, F.; Spearman, P.; Fichou, D.; Nogues, C.; Pan, X.; Garnier, F. Adv. Mater. 1996, 8, 242.

[5] Yui, K.; Ishida, H.; Aso, Y.; Otsubo, T.; Ogura, F. Chem. Lett. 1987, 16, 2339.

[6] Yui, K.; Aso Y.; Otsubo, T.; Ogura, F. Bull. Chem. Soc. Jpn. 1989, 62, 1539.

[7] Yui, K.; Ishida, H.; Aso, Y.; Otsubo T.; Ogura, F.; Kawamoto, A.; Tanaka, J. Bull. Chem. Soc. Jpn. 1989, 62, 1547.

[8] Higuchi, H.; Nakayama, T.; Koyama, H.; Ojima, J.; Wada, T.; Sasabe, H. Bull. Chem. Soc. Jpn. 1995, 68, 2363.

[9] Uno, M.; Seto, K.; Takahashi, S. J. Chem. Soc., Chem. Commun. 1984, 932.

[10] Pappenfus, T. M.; Hermanson, B. J.; Helland, T. J.; Lee, G. G. W.; Drew, S. M.; Mann, K. R.; McGee, K. A.; Rasmussen, S. C. Org. Lett. 2008, 10, 1553.

[11] Getmanenko, Y. A.; Purcell, T. A.; Hwang, D. K.; Kippelen, B.; Marder, S. R. J. Org. Chem. 2012, 77, 10931.

[12] Pappenfus, T. M.; Chesterfield, R. J.; Frisbie, C. D.; Mann, K. R.; Casado, J.; Raff, J. D.; Miller, L. L. J. Am. Chem. Soc. 2002, 124, 4184 .

[13] Chesterfield, R. J.; Newman, C. R.; Papperfus, T. M.; Ewbank, P. C.; Haukaas, M. H.; Mann, K. R.; Miller, L. L.; Frisbie, C. D. Adv. Mater. 2003, 15, 1278.

[14] Janzen, D. E.; Burand, M. W.; Ewbank, P. C.; Pappenfus, T. M.; Higuchi, H.; Filho, D. A. S.; Young, V. G.; Brédas, J. L.; Mann, K. R. J. Am. Chem. Soc. 2004, 126, 15295.

[15] Takahashi, T.; Matsuoka, K.; Takimiya, K.; Otsubo, T.; Aso, Y. J. Am. Chem. Soc. 2005, 127, 8928.

[16] Berlin, A.; Grimoldi, S.; Zotti, G.; Osuna, R. M.; Delgado, M. C. R.; Ortiz, R. P.; Casado, J.; Hernández, V.; Navarrete, J. T. L. J. Phys. Chem. B 2005, 109, 22308.

[17] Handa, S.; Miyazaki, E.; Takimiya, K.; Kunugi, Y. J. Am. Chem. Soc. 2007, 129, 11684.

[18] Wu, Q.-H.; Ren, S.-D.; Wang, M.; Qiao, X.-L.; Li, H.-X.; Gao, X.-K.; Yang, X.-D.; Zhu, D.-B. Adv. Funct. Mater. 2013, 23, 2277.

[19] Nakano, M.; Osaka, I.; Takimiya, K. J. Mater. Chem. C 2015, 3 , 283.
[20] Yamamoto, K.; Ie, Y.; Nitani, M.; Tohnai, N.; Kakiuchi, F.; Zhang, K.; Pisula, W.; Asadi, K.; Blom, P. W. M.; Aso, Y. J. Mater. Chem. C 2018, 6, 7493 .

[21] Handa, S.; Miyazaki, E.; Takimiya, K. Chem. Commun. 2009, 3919.

[22] Kunugi, Y.; Takimiya, K.; Toyoshima, Y.; Yamashita, K.; Aso, Y.; Otsubo, T. J. Mater. Chem. 2004, 14, 1367.

[23] Qiao, Y.-L.; Zhang, J.; Xu, W.; Zhu, D.-B. J. Mater. Chem. 2012 22, 5706.

[24] Xiong, Y.; Tao, J.-W.; Wang, R.-H.; Qiao, X.-L.; Yang, X.-D.; Wang, D.-L.; Wu, H.-Z.; Li, H.-X. Adv. Mater. 2016, $28,5949$.

[25] Qiao, Y.-L.; Guo, Y.-L.; Yu, C.-M.; Zhang, F.-J.; Xu, W.; Liu, Y.-Q.; Zhu, D.-B. J. Am. Chem. Soc. 2012, 134, 4084.

[26] Zhong, H.-L.; Smith, J.; Rossbauer, S.; White, A. J. P. Adv. Mater. 2012, 24, 3205.

[27] Wang, C.; Zang, Y.-P.; Qin, Y.-K.; Zhang, Q., Sun, Y.-H.; Di, C.-A.; Xu, W.; Zhu, D.-B. Chem.-Eur. J. 2014, 20, 13755.

[28] Wang, C.; Qin, Y.-K.; Sun, Y.-H.; Guan, Y.-S.; Xu, W.; Zhu, D.-B. ACS Appl. Mater. Interfaces 2015, 7, 15978.

[29] Ray, S.; Sharma, S.; Salzner, U.; Patil, S. J. Phys. Chem. C 2017, $121,16088$.

[30] Ren, L.-B.; Yuan, D.-F.; Zhu, X.-Z. Chem.-Asian J. 2019, 14, 1717.

[31] Zhang, C.; Zang, Y.-P.; Gann, E.; McNeill, C. R.; Zhu, X.-Z.; Di, C.-A.; Zhu, D.-B. J. Am. Chem. Soc. 2014, 136, 16176.

[32] Zhang, C.; Zang, Y.-P.; Zhang, F.-J.; Diao, Y.; McNeill, C. R.; Di, C.-A.; Zhu, X.-Z.; Zhu, D.-B. Adv. Mater. 2016, $28,8456$.

[33] Zhang, C.; Yuan, D.-F.; Wu, H.; Gann, E.; Thomsen, L.; McNeill, C.-R.; Di, C.-A.; Zhu, X.-Z.; Zhu, D.-B. J. Mater. Chem. C 2017, 5, 1935.

[34] Zhang, C.; Rivero, S. M.; Liu, W.-Y.; Casanova, D.; Zhu, X.-Z.; Casado, J. Angew. Chem., Int. Ed. 2019, 58, 11291.

[35] Chonan, T.; Takahashi, K. Bull. Chem. Soc. Jpn. 2004, 77, 1487.

[36] Kozaki, M.; Sugimura, K.; Ohnishi, H.; Okada, K. Org. Lett. 2006, 8,5235 .

[37] Kashiki, T.; Miyazaki, E.; Takimiya, K. Chem. Lett. 2009, 38, 568.

[38] Yanai, N.; Mori, T.; Shinamura, S.; Osaka, I.; Takimiya, K. Org. Lett. 2014, 16, 240.

[39] Mori, T.; Yanai, N.; Osaka, I.; Takimiya, K. Org. Lett. 2014, 16 , 1334.

[40] Wu, Q.-H.; Li, R..; Hong, W.; Li, H.-X.; Gao, X.-K.; Zhu, D.-B. Chem. Mater. 2011, 23, 3138.

[41] Li, J.; Qiao, X.-L.; Xiong, Y.; Li, H.-X.; Zhu, D.-B. Chem. Mater. 2014, 26, 5782.

[42] Xia, D.-B.; Keerthi, A.; An, C.-B.; Baumgarten, M. Org. Chem. Front. 2017, 4, 18.

[43] Jiang, H.; Oniwa, K.; Xu, Z. Q.; Bao, M.; Yamamoto, Y.; Jin, T.-N. Bull. Chem. Soc. Jpn. 2017, 90, 789.

[44] Suzuki, Y.; Miyazaki, E.; Takimiya, K. J. Am. Chem. Soc. 2010, 132, 10453.

[45] Jiang, H.; Zhang, L.; Cai, J.-F.; Ren, J.-H.; Cui, Z.-H.; Chen, W.-G. Dyes Pigm. 2018, 151, 363.

[46] Ren, L.-B.; Liu, F.; Shen, X.-X.; Zhang, C.; Yi, Y.-P.; Zhu, X.-Z. J. Am. Chem. Soc. 2015, 137, 11294.

[47] Huang, C.-B.; Chen, H.; Li, F.-Q; An, S.-Y. Chin. J. Org. Chem. 2019, 39, 2467 (in Chinese). (黄池宝, 陈会, 李福琴, 安思雅, 有机化学, 2019, 39, 2467.)

[48] Wang, J.-J.; Qi, S.-L.; Du, J.-S.; Yang, Q.-B.; Song, Y.; Li, Y.-X Chem. J. Chin. Univ. 2019, 40, 1397 (in Chinese). (王金金，戚少龙，杜建时，杨清彪，宋岩，李耀先，高等学校化 学学报, 2019, 40, 1397.)

[49] Suzuki, Y.; Shimawaki, M.; Miyazaki, E.; Osaka, I.; Takimiya, K. Chem. Mater. 2011, 23, 795.

[50] Ishii, A.; Horikawa, Y.; Takaki, I.; Shibata, J.; Nakayama, J.; Hoshino, M. Tetrahedron Lett. 1991, 32, 4313.

[51] Takeda, T.; Akutagawa, T. J. Org. Chem. 2015, 80, 2455.

[52] Kawase, T.; Ueno, N.; Oda, M. Tetrahedron Lett. 1992, 33, 5405.

[53] Bhattacharyya, K.; Dey, D.; Datta, A. J. Phys. Chem. C 2019, 123, 4749.

[54] Mazaki, Y.; Murata, S.; Kobayashi, K. Tetrahedron Lett. 1991, 32, 
4367.

[55] Kawata, S.; Pu, Y. J.; Saito, A.; Kurashige, Y.; Beppu, T.; Katagiri, H.; Hada, M.; Kido, J. Adv. Mater. 2016, 28, 1585.

[56] Takahashi, K.; Suzuki, T. J. Am. Chem. Soc. 1989, 111, 5483.

[57] Kan, Z.-P.; Colella, L.; Canesi, E. V.; Lerario, G.; Kumar, R. S. S.; Bonometti, V.; Mussini, P. R.; Cavallo, G.; Terraneo, G.; Pattanasattayavong, P.; Anthopoulos, T. D.; Bertarelli, C.; Keivanidis, P. E. Sol. Energy Mater. Sol. Cells 2014, 120, 37.

[58] Agostinelli, T.; Caironi, M.; Natali, D.; Sampietro, M.; Dassa, G.; Canesi, E. V.; Bertarelli, C.; Zerbi, G.; Cabanillas-Gonzalez, J.; Silvestri, S. D.; Lanzani, G. J. Appl. Phys. 2008, 104, 114508.

[59] Fazzi, D.; Canesi, E. V.; Negri, F.; Bertarelli, C.; Castiglioni, C. ChemPhysChem 2010, 11, 3685 .

[60] Canesi, E. V.; Fazzi, D.; Colella, L.; Bertarelli, C.; Castiglioni, C. J. Am. Chem. Soc. 2012, 134, 19070.

[61] Colella, L.; Brambilla, L.; Nardone, V.; Parisini, E.; Castiglioni, C.; Bertarelli, C. Phys. Chem. Chem. Phys. 2015, 17, 10426.

[62] Francesco, T.; Colella, L.; Maghsoumi, A.; Martí-Rujas, J.; Parisini, E.; Tommasini, M.; Bertarelli, C.; Barbon, A. J. Phys. Chem. C 2016, 120, 5732.

[63] Tormos, G. V.; Belmore, K. A.; Cava, M. P. J. Am. Chem. Soc. 1993, 115,11512 .

[64] Hwang, H.; Khim, D.; Yun, J. M.; Jung, E.; Jang, S. Y.; Jang, Y. H.; Noh, Y. Y.; Kim, D. Y. Adv. Funct. Mater. 2015, 25, 1146.

[65] Ren, L.-B.; Fan, H.-J.; Huang, D.-Z.; Yuan, D.-F.; Di, C.-A.; Zhu, X.-Z. Chem.-Eur. J. 2016, 22, 17136.

[66] Deng, Y.-F.; Sun, B.; Quinn, J.; He, Y.-H.; Ellard, J.; Guo, C.; Li, Y.-N. RSC Adv. 2016, 6, 45410.

[67] Deng, Y.-F.; Quinn, J.; Sun, B.; He Y.-H.; Ellard, J.; Li, Y.-N. RSC Adv. 2016, 6, 34849

[68] Deng, Y.-F.; Sun, B.; He, Y.-H.; Quinn, J.; Guo, C.; Li, Y.-N. Angew. Chem., Int. Ed. 2016, 55, 3459.

[69] Kozaki, M.; Isoyama, A.; Akita, K.; Okada, K. Org. Lett. 2005, 7, 115.

[70] Kozaki, M.; Isoyama, A.; Okada, K. Tetrahedron Lett. 2006, 47, 5375 .

[71] Ay, E.; Furukawa, S.; Nakamura, E. Org. Chem. Front. 2014, 1,
988.

[72] Rudebusch, G. E.; Fix, A. G.; Henthorn, H. A.; Vonnegut, C. L.; Zakharov, L. N.; Haley, M. M. Chem. Sci. 2014, 5, 3627.

[73] Shi, X.-L.; Burrezo, P. M.; Lee, S.-S.; Zhang, W.-H.; Zheng, B.; Dai, G.-L.; Chang, J.-J.; Navarrete, J. T. L.; Huang, K. W.; Kim, D. H.; Casado, J.; Chi, C. Y. Chem. Sci. 2014, 5, 4490.

[74] Shi, X.-L.; Lee, S.-S.; Son, M.-J.; Zheng, B.; Chang, J.-J.; Jing, L.-Z.; Huang, K.-W.; Kim, D.-H.; Chi, C.-Y. Chem. Commun. 2015, $51,13178$.

[75] Shi, X.-L.; Quintero, E.; Lee, S.-S.; Jing, L.-Z.; Herng, T. S.; Zheng, B.; Huang, K. W.; Navarrete, J. T. L.; Ding, J.; Kim, D. H.; Casado, J.; Chi, C.-Y. Chem. Sci. 2016, 7, 3036.

[76] Tan, J.-H.; Huo, Y.-P.; Cai, N.; Ji, S.-M.; Li, Z.-Z.; Zhang, L. Chin J. Org. Chem. 2017, 37, 2457 (in Chinese). (谭继华, 霍延平, 蔡宁, 籍少敏, 李宗植, 张力, 有机化学, 2017, 37, 2457.)

[77] Inoue, S.; Mikami, S.; Aso, Y.; Otsubo, T.; Wada, T.; Sasabe, H. Synth. Met. 1997, 84, 395.

[78] Holzmüller, F.; Gräßler, N.; Sedighi, M.; Müller, E.; Knupfer, M.; Zeika, O.; Vandewal, K.; Koerner, C.; Leo, K. Org. Electron. 2017, 45, 198.

[79] Takahashi, T.; Takimiya, K.; Otsubo, T.; Aso, Y. Org. Lett. 2005, 7, 4313.

[80] Guegano, X.; Kanibolotsky, A. L.; Blum, C.; Mertens, S. F. L.; Liu, S. X.; Neels, A.; Hagemann, H.; Skabara, P. J.; Leutwyler, S.; Wandlowski, T.; Hauser, A.; Decurtins, S. Chem.-Eur. J. 2009, 15, 63.

[81] Zhang, Q.-Q.; Jiang, K.-J.; Huang, J.-H.; Zhao, C.-W.; Zhang, L.-P.; Cui, X.-P.; Su, M.-J.; Yang, L.-M.; Song, Y.-L.; Zhou, X.-Q. J. Mater. Chem. A 2015, 3, 7695.

[82] Gräßler, N.; Wolf, S.; Holzmüller, F.; Zeika, O.; Vandewal, K.; Leo, K. Eur. J. Org. Chem. 2019, 845 .

[83] Cai, J.-F.; Chen, W.-G.; Cui, Z.-H.; Jiang, H. J. Text. Res. 2018, 39, 81 (in Chinese). (蔡金芳，陈维国，崔志华，江华，纺织学报, 2018, 39, 81.)

[84] Jiang, H.; Hu, Q.; Cai, J.-F.; Cui, Z.-H.; Zheng, J.-H.; Chen, W.-G. Dyes Pigm. 2019, 166, 130.

(Zhao, C.) 